\title{
Waves and Instabilities in High Quality Adiabatic Flow in Microgap Channels
}

\author{
Avram Bar-Cohen, Caleb Holloway ${ }^{*}$, Ahmed Kaffel, Amir Riaz \\ Thermal Packaging of Electronic and Photonic Systems (TherPES), Department of \\ Mechanical Engineering, University of Maryland, College Park, MD 20742, USA
}

\section{Abstract}

Two-phase flow in microgap channels offers highly potent thermal management capability and is the foundation for the emerging "embedded cooling" paradigm of electronic cooling. While heat transfer and pressure drop in such flows are intimately tied to their distinct forms of vapor-liquid aggregation, insufficient attention has been paid to characterizing the wave patterns and sub-regimes in high-quality microgap channel flow. The present visualization study focuses on two-phase flow in an adiabatic $184 \mu \mathrm{m}$ microgap channel operating at three mass fluxes of FC-72: 220,420 , and $620 \mathrm{~kg} / \mathrm{m}^{2}-\mathrm{s}$, with flow qualities ranging from approximately $40 \%$ to $90 \%$. As predicted by a modified Taitel-Dukler flow regime map, annular flow is found to be the dominant flow regime for the present microgap configuration. Within the annular flow regime, unique 3-D wave patterns are observed at the liquid-vapor interface. The wavelength of these interfacial waves is observed to decrease with increasing flow quality and mass flux. Linear stability analysis of the liquid-vapor interface is found to yield strong agreement in predicted wavelength and wave growth rate distribution with the experimental results.

Keywords:

Two-phase Annular flow, Shear-driven film, Interfacial wave patterns, Interfacial instability, Microgap

\section{Introduction}

Thermal management of high-powered electronics has become increasingly challenging with escalating power dissipation densities and demanding design requirements. In addition to power dissipations in excess of $100 \mathrm{~W}$ for chips targeted to operate below $100^{\circ} \mathrm{C}$, sub-millimeter microprocessor hot spots can exceed heat fluxes of $1 \mathrm{~kW} / \mathrm{cm}^{2}$. Even more challenging, HEMT GaN transistors, used in power amplifiers, can generate heat fluxes that are typically greater than $10 \mathrm{~kW} / \mathrm{cm}^{2}$ and limited to gate temperatures of $150^{\circ} \mathrm{C}-200^{\circ} \mathrm{C}$ to ensure reliable, efficient, and stable RF operation (Kopp et al., 2000; Bar-Cohen et al., 2011).

A dielectric coolant, flowing in a microgap channel while undergoing phase change, is a promising thermal solution for direct cooling of such high-powered electronics (Bar-Cohen et al., 2012a; Kim et al., 2010; Alam et al., 2012; Kim et al., 2008; Bar-Cohen et al., 2011; Bar-Cohen et al., 2009; Bar-Cohen et al., 2012b; Yen et al., 2003; Mastrullo et al., 2012; Cortina-Diaz and Schmidt, 2006; Yang and Fujita, 2004) and offers the inherent benefits of high heat transfer coefficients, small temperature gradients, excellent hot spot mitigation, and low pumping power requirements. However, the thermofluid characteristics, thermal performance, and limits of two-phase microgap

* Corresponding author. Tel.: 301-405-8223

Email address: calebh@umd.edu 
channels, especially in the favorable domain of high vapor fractions or flow qualities, are poorly understood. Widespread implementation of evaporative microgap cooling, and by extension inter-chip as well as microchannel cooling (Bar-Cohen et al., 2011) is contingent on a better understanding and prediction of the fundamental thermo-physics of high quality, two-phase flow in microgaps.

Extensive infrared thermography of an evaporatively-cooled, asymmetricallyheated microgap channel has revealed the existence of previously unobserved, large amplitude, moderate frequency wall temperature fluctuations in high-quality, annular flow (Bar-Cohen et al., 2012b). The authors postulated that these fluctuations were caused by shear-driven instabilities, producing localized and periodic liquid film rupture, with associated wall temperature spikes, followed by liquid quenching and wall cooldown. As the size and duration of the ruptured film, or local dryout, zones grow, the average heat transfer coefficient decreases from values associated with thin film evaporation towards values associated with vapor convection. In an effort to shed light on such shear-driven instabilities in annular flow, Kabov et al. (2011) empirically studied the liquid-vapor interface in an adiabatic, $40 \mathrm{~mm} \times 80 \mathrm{~mm} \times 2 \mathrm{~mm}$ high channel, containing two independently injected fluid streams - nitrogen vapor and FC-72 liquid. The thin-film interface, visually observed and characterized using the Phase Shifting Schlieren (PSS) technique, was found to display a variety of "deformations," including five distinct sub-regimes: (1) cells, (2) structures, (3) 2-D waves, (4) 3-D waves, and (5) film rupture. At low liquid and vapor Reynolds numbers, cells and structure were observed, consisting of a smooth film with weak deformations. With increasing gas velocity, 2-D waves emerge; the 2-D wavelength continues to decrease with increasing gas velocity until transitioning into 3-D waves with a broad range of wavelengths. At low liquid Reynolds numbers and sufficiently large gas Reynolds numbers, the liquid film ruptures and is torn from the microgap surface. Similar results for a smaller microgap channel were recently presented by Houshmand and Peles (2013), who studied the sheardriven liquid-gas interface by separately injecting water and nitrogen into a $1.5 \mathrm{~mm}$ wide, $220 \mu \mathrm{m}$ deep microgap channel. With increasing gas velocity, the liquid-gas interface progressed from smooth to 2-D to 3-D waves.

The present work examines the waves and instabilities that occur in high quality adiabatic flow in a microgap channel with the help of experimental observations and linear stability analysis. Unlike in the Kabov et al. (2011) and Houshmand and Peles (2013) studies, and as a closer representation of an actual microgap cooling configuration, for a specified liquid flow rate, heat addition is used to create a vapor stream - of the same species as the liquid stream - and set the vapor fraction in the channel. To enable the observation of adiabatic channel behavior, an upstream evaporator is used to control the thermodynamic flow quality of an imposed liquid flow rate and no additional heat transfer occurs in the channel itself. The upstream evaporator, thus, creates various ratios of liquid and vapor mass flux and superficial velocity and establishes the shear-driven flow through the adiabatic microgap test section. It is to be noted that this same configuration will then be used for a subsequent diabatic (heated) microgap channel study, with a saturated liquid inlet and a progressively higher quality as the fluid traverses the microgap channel.

The linear stability analysis is carried out to highlight the relevant physical mechanisms that govern the hydrodynamic stability behavior. This approach is motivated 
by the observation of interfacial waves, in particular for high quality flows, that appear to exist in a state of nonlinear saturation where the wave amplitude remains relatively stable with the progression of waves down the microgap channel. The linear stability analysis thus helps identify the dominant wave structure that is expected to persist as the wave amplitude evolves from infinitesimal perturbations, within the regime of linear growth, to fully developed nonlinear behavior. This information is valuable in the context of the analysis of the spatial development of thermal fluctuations that are expected to be correlated with the wave structure produced by hydrodynamic instability. Our experimental characterization of interfacial wave patterns for adiabatic flow within the microgap channel indicates good agreement with the spectrum of unstable wavelengths predicted by the linear stability analysis. As a result, it becomes possible to rigorously investigate physical mechanisms that set the wavelength for a wide range of flow conditions.

The study is organized as follow. Section 3 describes the experimental setup and procedure. Section 4 presents a detailed characterization of visual interfacial patterns within the microgap channel. Linear stability analysis corresponding to experimental flow conditions is presented in Section 5. This is followed by the summary of findings and conclusions in Section 6.

\section{Experimental Setup and Procedure}

\subsection{Apparatus}

A schematic of the flow loop used for the present study is shown in Fig. 1. The coolant (FC-72) is stored in an atmospheric reservoir and pumped with a Fluid-o-Tech magnetically coupled gear pump and DC motor. The flow rate is measured with a Kobold DPM pelton wheel flow sensor and the liquid temperature with an Omega T-type thermocouple probe, before it proceeds into a resistive coil heater (evaporator). The evaporator pre-heats the subcooled liquid to a targeted level of thermodynamic quality based on an energy balance calculation. Power is measured and applied to the evaporator with a BK Precision DC power supply. Before the two-phase flow enters the test section, the pressure and temperature are measured with a Gems 2200 pressure transducer and an Omega T-type thermocouple probe, respectively. The two-phase flow then proceeds through the microgap test section and visually captured using a Nikon D2x SLR with a Nikkor PC Micro $85 \mathrm{~mm}$ lens and Speedlight flash. The pressure drop across the channel is measured with a Setra 230 pressure transducer and the outlet temperature with an Omega T-type thermocouple probe. A Flat Plate liquid-to-liquid brazed plate heat exchanger condenses the two-phase mixture before it flows back into the reservoir. The flow loop consists of Swagelock stainless steel plumbing and a National Instruments CompactDAQ system with a NI 9214 thermocouple, NI 9205 analog voltage, and NI 9203 analog current module, of which are used to measure temperature (thermocouples), pressure, and flow rate.

The adiabatic visualization of the micro-gap test section was performed for three mass fluxes, $G=220,420$, and $620 \mathrm{~kg} / \mathrm{m}^{2}$-s, corresponding to flow rates of $0.5,1.0$, and $1.5 \mathrm{~mL} / \mathrm{s}$. For a given mass flux, the desired thermodynamic quality entering the adiabatic micro-gap channel was controlled using the pre-heater and varied from 
approximately 40 to $85 \%$, in four steps. Visualization was performed for each distinct mass flux and thermodynamic quality case when a quasi-steady state condition was reached.

\subsection{Test Section}

The microgap test section is shown in Fig. 2. The lower portion of the test section consists of a solid piece of copper with the microgap and reservoirs milled into the upward-facing surface. The microgap channel is $35 \mathrm{~mm}$ long, $20 \mathrm{~mm}$ wide, and has a milled gap depth of $184 \mu \mathrm{m}$. Thermocouple holes, $1 \mathrm{~mm}$ in diameter and $1 \mathrm{~mm}$ below the microgap's surface, are drilled laterally into the copper substrate and meet at the channel's centerline. The upper portion of the channel is confined with a polycarbonate cover and hermetically face-sealed with an o-ring. The upper cover also serves as an optical window for visualization of the two-phase microgap flow.

\subsection{Data Reduction}

The average thermodynamic quality, $x$, is calculated using the following energy balance:

$x=\frac{\left[s Q-\dot{m}_{l} C_{p, l}\left(T_{s a t}(P)-T_{\text {res. }}\right)\right]}{\dot{m}_{l} h_{l v}}$

The pressure, $P$, is measured at the inlet of the microgap and the temperature of the single-phase FC-72 liquid from the reservoir, $T_{\text {res }}$, is measured right before the inline heater, as shown in Fig. 1. The estimated RSS uncertainty in thermodynamic quality ranges from 2.6 to $6.6 \%$, where the largest uncertainty occurs at the lowest flow rate.

The heat loss, $Q_{\text {loss }}$, and conversion efficiency, $\varsigma$, is calculated from equations (2) and (3), respectively. The heat loss is determined experimentally from a single-phase sensible heat balance for the average wall temperature range encountered in the twophase tests and varies linearly with the average temperature difference of the channel. The average wall temperature, $T_{\text {wall, avg }}$, is equal to the average saturation temperature of the fluid within the channel and the ambient temperature, $T_{\infty}$, is at a constant temperature of $23^{\circ} \mathrm{C}$. Overall, the heat losses are minimal and have a small effect on the computed parameters; this is expected, as the apparatus is extensively insulated.

$$
\begin{aligned}
& Q_{\text {loss }}=0.052\left(T_{\text {wall }, \text { avg }}-T_{\infty}\right) \\
& \varsigma=\frac{Q-Q_{\text {loss }}}{Q}
\end{aligned}
$$

The superficial liquid velocity, superficial vapor velocity, and mass flux is calculated with equations (4), (5), and (6), respectively: 
$U_{s l}=\frac{(1-x) G}{\rho_{l}}$

$U_{s v}=\frac{x G}{\rho_{v}}$

$G=\frac{\dot{m}_{l}}{A_{c s}}=\frac{\dot{m}_{l}}{w \delta}$

In equation (6), $w$ is the width and $\delta$ the height of the microgap. The estimated RSS uncertainty in superficial liquid velocity, vapor velocity, and mass flux is 0.0066 to $0.012 \mathrm{~m} / \mathrm{s}, 0.68$ to $1.5 \mathrm{~m} / \mathrm{s}$, and 16 to $25 \mathrm{~kg} / \mathrm{m}^{2}-\mathrm{s}$, respectively. A summary of the uncertainty in experimental parameters is tabulated in Table 1.

\subsection{Experimental Test Conditions, Fluid Properties, and Adiabatic Flow Regime Map}

The experimental test conditions and corresponding FC-72 fluid properties are tabulated in Table 2. The local saturation temperature of FC-72 within the microgap is highly dependent on the local pressure; accordingly, the density, viscosity, and surface tension are evaluated at the average saturation temperature, of which corresponds to the average pressure of the microgap.

The test conditions evaluated in the microgap test section are plotted on a TaitelDukler flow regime map, Fig. 3, with a modified Ullman-Brauner intermittent-to-annular boundary and the bubbly-to-intermittent transition as proposed by Rahim et al. (2011) and given by equations (7) and (8), respectively:

$U_{s l}=\frac{1-\left(\varepsilon_{v}\right)_{c r i t}}{\left(\varepsilon_{v}\right)_{c r i t}} U_{s v},\left(\varepsilon_{v}\right)_{c r i t}=0.67$

$U_{s v}=\frac{6.2\left[\gamma g\left(\rho_{l}-\rho_{v}\right)\right]^{1 / 4}}{\rho_{v}^{1 / 2}}$

For a given mass flux, the loci of experimental cases progress from left to right with increasing thermodynamic quality and superficial vapor velocity. The flow regime map suggests that the vast majority of cases are in the annular regime, as planned for this experimental study, with the lowest mass flux and thermodynamic quality case appearing to straddle the intermittent-to-annular transition.

\section{Visualization and Discussion}

\subsection{Interfacial Deformations and Waves}

The large slip velocities and shear-induced flow, which develop in high-quality, annular two-phase flow, as shown in the flow regime map of Fig. 3, have been found to lead to a progression from smooth to 2-D to 3-D waves at the liquid-vapor interface, and 
ultimately film rupture, as the quality increases (Kabov et al., 2011; Houshmand and Peles, 2013). The present work examines the interfacial deformations and structures that occur in high quality adiabatic flow in a microgap channel in which heat addition is used to create a vapor stream - of the same species - and set the vapor fraction in the channel. Results obtained, by varying the heat input of an upstream evaporator to control the thermodynamic flow quality in the $184 \mu \mathrm{m}$ microgap channel, will be presented sequentially for mass fluxes of $220 \mathrm{~kg} / \mathrm{m}^{2}-\mathrm{s}, 420 \mathrm{~kg} / \mathrm{m}^{2}-\mathrm{s}$, and $620 \mathrm{~kg} / \mathrm{m}^{2}-\mathrm{s}$. For each mass flux, visualization is captured at four separate flow qualities, ranging from approximately $40 \%$ to $85 \%$.

\subsubsection{Adiabatic Visualization: $F C-72, G=220 \mathrm{~kg} / \mathrm{m}^{2}-\mathrm{s}, \delta=184 \mu \mathrm{m}$}

Visualization of the lowest mass flux case, $220 \mathrm{~kg} / \mathrm{m}^{2}-\mathrm{s}$, is shown in Fig. 4, starting with 39\% quality in Fig. 4(a), corresponding to a superficial vapor and liquid velocity of $6.6 \mathrm{~m} / \mathrm{s}$ and $0.082 \mathrm{~m} / \mathrm{s}$, progressing to $54 \%$ quality in Fig. 4(b), with a superficial vapor and liquid velocity of $8.6 \mathrm{~m} / \mathrm{s}$ and $0.058 \mathrm{~m} / \mathrm{s}$. The visualization shown in Fig. 4(b) persists at a flow quality of 66\%, as shown in Fig. 4(c), corresponding to a superficial vapor and liquid velocity of $11 \mathrm{~m} / \mathrm{s}$ and $0.043 \mathrm{~m} / \mathrm{s}$. Visualization for the lowest mass flux case then concludes at a flow quality of 78\%, Fig. 4(d), where the superficial vapor and liquid velocity is $13 \mathrm{~m} / \mathrm{s}$ and $0.027 \mathrm{~m} / \mathrm{s}$.

For the lowest flow quality of 39\%, shown in Fig. 4(a), the two-phase flow is on the verge of transitioning from intermittent to annular flow. Vapor bubbles near the channel inlet, shown in the left portion of Fig. 4(a), are small relative to the lateral width of the channel but with increasing axial distance, grow in size as they interact and coalesce with neighboring bubbles. These growing vapor bubbles eventually occupy the entire width of the channel, as shown in the middle of Fig. 4(a). When the large vapor bubbles reach the reservoir at the channel exit, shown in the right portion of Fig. 4(a), the confined vapor is released and 3-D waves appear at the liquid-vapor interface.

As the flow quality increases from 39\% in Fig. 4(a) to 54\% in Fig. 4(b), annular flow dominates the entire length of the channel and 3-D waves appear at the liquid-vapor interface. The 3D waves are shaped with large and smooth radii, but with considerable variation in width, overlap, and wavelength. As the flow quality increases to $66 \%$, Fig. 4(c), the 3-D wave pattern persists but with a noticeable reduction in wave amplitude. However, at a flow quality of 78\%, shown in Fig. 4(d), the liquid film is very thin and, consequently, the curvature of the liquid-vapor interface less pronounced. While waves are still observed at the liquid-vapor interface, they consist of a continuous and wavy spanwise front with both smaller and more consistently grouped wavelengths.

It should be noted that the observed waves belong to the upper film, of which is in contact with the upper polycarbonate wall. Due to gravity, it's expected that the upper film is slightly thinner than the bottom film and that the surface roughness and wetting characteristics of the copper and polycarbonate surfaces may be slightly different. However, the importance of the surface roughness and wetting characteristics is only significant for very thin films (on the order of the surface roughness) and in the case of de-wetting/film rupture (of which is not observed in this study), respectively.

A Matlab algorithm is used to measure the number of pixels between the upper film wave crests (in the streamwise direction) and is then converted to a physical distance 
based on the known length of the channel. For each individual case, the mean and standard deviation of wavelengths is computed from a sample size of approximately 30,000 measurements, of which is measured in the second half of the channel (i.e., the last $17.5 \mathrm{~mm}$ of the microgap) in order to negate the effects of developing flow at the entrance of the microgap. Given the limited resolution of the images, the uncertainty in the measured wavelength is estimated to equal $\pm 25 \mu \mathrm{m}(2-13 \%)$. A tabulation of the case conditions and measured wavelengths for the $220 \mathrm{~kg} / \mathrm{m}^{2}-\mathrm{s}$ mass flux is provided in Table 3, where a slight reduction in the average wavelength with increasing flow quality may be seen.

But, more interestingly, Table 3 reveals a significant drop in the standard deviation of wavelengths from a quality of $66 \%$ to $78 \%$, reducing from $0.73 \mathrm{~mm}$ to $0.48 \mathrm{~mm}$, respectively. This sudden reduction in standard deviation and tighter grouping of wavelengths corresponds to the emergence of "continuous" waves, as shown in Fig. 4(d).

\subsubsection{Adiabatic Visualization: $F C-72, G=420 \mathrm{~kg} / \mathrm{m}^{2}-\mathrm{s}, \delta=184 \mu \mathrm{m}$}

Visualization of the moderate mass flux case, $420 \mathrm{~kg} / \mathrm{m}^{2}-\mathrm{s}$, is shown in Fig. 5 , starting at $43 \%$ quality in Fig. 5(a), with a superficial vapor and liquid velocity of $13 \mathrm{~m} / \mathrm{s}$ and $0.14 \mathrm{~m} / \mathrm{s}$, respectively, continuing to $72 \%$ quality in Fig. 5(b), with a superficial vapor and liquid velocity of $20 \mathrm{~m} / \mathrm{s}$ and $0.071 \mathrm{~m} / \mathrm{s}$, respectively, and reaching 85\% quality in Fig. 5(c), with a corresponding vapor and liquid velocity of $24 \mathrm{~m} / \mathrm{s}$ and $0.035 \mathrm{~m} / \mathrm{s}$.

At the lowest flow quality of $43 \%$, shown in Fig. 5(a), the entire channel is occupied by annular flow; but unlike the $220 \mathrm{~kg} / \mathrm{m}^{2}$-s mass flux case, intermittent flow is not observed. The waves at the liquid-vapor interface are no longer smooth in appearance, as was the case for the $220 \mathrm{~kg} / \mathrm{m}^{2}$-s mass flux, but chaotic. The film's features are very small, scaly, and lack any sort of apparent order. As the quality increases to $56 \%$, this observed character was found to persist and remain unchanged. Assuming the vapor velocity is proportional to mass flux, this sudden change in the films appearance compared to the lower mass flux case, may be the consequence of a now turbulent vapor core, resulting in unsteady, but also larger, interfacial shear forces. At a quality of $72 \%$, shown in Fig. 5(b), "continuous" waves randomly emerge amidst the chaotic structure. These waves share the same characteristics of those previous observed and described in Fig. 4(d) but the crests appear to be larger in amplitude and not as well defined. As the flow quality increases to $85 \%$, the scaly liquid film disappears and the "continuous" waves consume the entire channel, as shown in Fig. 5(c).

A tabulation of the case conditions and measured wavelengths for the $420 \mathrm{~kg} / \mathrm{m}^{2}$-s mass flux is provided in Table 4 . The average wavelength gradually decreases from $0.40 \mathrm{~mm}$ to $0.34 \mathrm{~mm}$ as the quality increase from 43 to $72 \%$, respectively. At a quality of $85 \%$, the average wavelength abruptly drops to $0.23 \mathrm{~mm}$, along with a $50 \%$ reduction in standard deviation; this transition corresponds to the emergence "continuous" waves, as shown in Fig. 5(c). For a comparable flow quality, the average wavelength is roughly 2-3 times smaller than those measured for the lower $220 \mathrm{~kg} / \mathrm{m}^{2}$-s mass flux cases. 


\subsubsection{Adiabatic Visualization: $F C-72, G=620 \mathrm{~kg} / \mathrm{m}^{2}-\mathrm{s}, \delta=184 \mu \mathrm{m}$}

Visualization of the interfacial structures associated with the largest mass flux, $620 \mathrm{~kg} / \mathrm{m}^{2} \mathrm{~s}$, is shown in Fig. 6, for a range of flow qualities. Fig 6(a) displays the interfacial structures for a $38 \%$ quality, corresponding to a superficial vapor and liquid velocity of $15 \mathrm{~m} / \mathrm{s}$ and $0.24 \mathrm{~m} / \mathrm{s}$, respectively. The appearance of the liquid-vapor interface at a $54 \%$ quality, with a superficial vapor and liquid velocity of $21 \mathrm{~m} / \mathrm{s}$ and $0.18 \mathrm{~m} / \mathrm{s}$, respectively, is shown in Fig 6(b), while Fig 6(c) displays the observed structures for a flow quality of $70 \%$, with a superficial vapor and liquid velocity of $25 \mathrm{~m} / \mathrm{s}$ and $0.12 \mathrm{~m} / \mathrm{s}$. The observed interfacial waves at $87 \%$ quality, corresponding to a superficial vapor and liquid velocity of $30 \mathrm{~m} / \mathrm{s}$ and $0.046 \mathrm{~m} / \mathrm{s}$, are similar to those shown in the third panel of Fig. 6.

As the mass flux increases from $420 \mathrm{~kg} / \mathrm{m}^{2}-\mathrm{s}$ to $620 \mathrm{~kg} / \mathrm{m}^{2}-\mathrm{s}$, the observed appearance is largely unaltered. At the lowest flow quality of 38\%, shown in Fig. 6(a), annular flow consistently extends along the entire length of the channel and, as before, consists of a chaotic, scaly, liquid-vapor interface. This appearance persists as the flow quality increases to $54 \%$. As the flow quality increases to $70 \%$ in Fig. 6(c), "continuous" waves emerge, as with the $420 \mathrm{~kg} / \mathrm{m}^{2}$-s case, and are visible, as well, at a flow quality of 87\%. These waves are not quite as well defined, or as "crisp", as those shown in Fig. 5(c) but the structure is similar.

A tabulation of the case conditions and measured wavelengths for the $620 \mathrm{~kg} / \mathrm{m}^{2}$-s mass flux is provided in Table 5. As was the case for the lower mass fluxes, the average wavelength decreases with increasing flow quality: from $0.42 \mathrm{~mm}$ at a quality of $38 \%$, to $0.21 \mathrm{~mm}$ at a quality of $87 \%$. For a similar quality, the average wavelength and standard deviation is only slightly smaller - on average - than those observed for the $420 \mathrm{~kg} / \mathrm{m}^{2}$-s mass flux.

\section{Linear Stability Analysis}

Interfacial waves observed in the experiments described above result from two basic mechanisms of hydrodynamic instability. First, velocity gradients normal to the mean flow direction give rise to a shear related mode of instability. In this case, the destabilizing inertial effects are balanced by viscous damping. Second, the viscosity difference between the liquid and the vapor phase leads to an interfacial mode of instability. Here, the discontinuity in normal viscous stress across the interface is balanced by both interfacial tension and viscous damping. In the context of linear stability analysis of nearly parallel channel flows, while the shear mode of instability occurs beyond a critical Reynolds number, the interfacial mode of instability exists independently of the Reynolds number, as long as the viscosity of the two fluids is unequal (Yih, 1963; Hooper and Boyd, 1985). For the specific range of Reynolds numbers encountered in our experimental study, the interfacial mode of instability is expected to exert a somewhat greater influence on the formation of interfacial waves, as discussed in section 5.2.

A linear stability analysis is used in this study to determine growth characteristics of small amplitude perturbations with respect to steady, fully developed two-phase annular flow in channels. In real systems, perturbations can arise from non-uniformities 
of inlet flow, equipment vibrations, and surface irregularities, leading to interfacial wavelike structures such as those observed in our experiments. The time required for transitioning from small amplitude perturbations to the experimentally observed wave profiles depends on the initial perturbation amplitude. In our experimental study, although the observed interfacial waves likely originate as finite amplitude perturbations, bypassing the linear regime, the wavelength is predicted reasonably well by the linear stability analysis, as discussed in section 5.3. This suggests that wavelength-coarsening mechanisms are relatively weak, particularly for the case of thinner liquid layers, such that the dominant waves do not undergo substantial variation in wavelength when transitioning from the linear to the saturated nonlinear state. Experimental observations also indicate that both the amplitude and wavelength do not change substantially as the waves move down the channel. Thus, we find it feasible to carry out a temporal stability analysis to identify the dominant spectral composition of interfacial waves.

\subsection{Governing Equations}

A two-dimensional planar system will be considered, as shown in Fig. 7, because the primary mode of instability is 2-D (Drazin and Reid, 1981; Drazin, 2002). The undisturbed flow is parallel to the $x$-axis, and the $y$-axis origin is at the undisturbed interface, as shown in Fig. 7. The velocity profiles in the liquid and vapor layers are assumed to be fully developed. Disturbances at the upper and lower interfaces are also assumed to develop independently because the liquid films are relatively thin compared with the channel height. Accordingly, the stability analysis is simplified by considering only the "symmetric" lower half of the channel, as shown in Fig. 7. The vapor core has viscosity $\mu_{v}$, density $\rho_{v}$, and depth $h_{v}$ while the lower liquid film is of viscosity $\mu_{l}$, density $\rho_{l}$, and depth $h_{l}$.

The basic velocity profile in the channel is obtained by solving for the fully developed velocity fields, $U_{v}$ and $U_{l}$, for the vapor and the liquid layers, respectively, to yield:

$U_{v}=\frac{1}{2 \mu_{v}} \frac{d p}{d x} y^{2}+c_{1} y+c_{2}, \quad U_{l}=\frac{1}{2 \mu_{l}} \frac{d p}{d x} y^{2}+c_{3} y+c_{4}$

where $d p / d x$ is the pressure gradient driving the entire flow of vapor and liquid. The dimensional constants $c_{1}, c_{2}, c_{3}, c_{4}$ are functions of $d p / d x$ in general (see Appendix) and are determined by applying the following boundary conditions:

$\left.U_{l}\right|_{y=-h_{l}}=\left.\frac{d U_{v}}{d y}\right|_{y=h_{v}}=0, \quad U_{l}(0)=U_{v}(0),\left.\quad \mu_{l} \frac{d U_{l}}{d y}\right|_{y=0}=\left.\mu_{v} \frac{d U_{v}}{d y}\right|_{y=0}$

The pressure gradient in Eq. (9) is obtained by considering the total mass flow rate, $\dot{M}=\dot{M}_{l}+\dot{M}_{v}$, where $\dot{M}_{l}$ and $\dot{M}_{v}$ represent the mass flow rate of the liquid and the vapor phase, respectively. The mass flow rate for each phase is obtained by integrating the corresponding velocity profiles in respective layers. The total mass flux, $G$, can then be related to the velocity profiles as: 
$G=\frac{2}{H} \int_{-h_{l}}^{0} \rho_{l} U_{l} d y+\frac{2}{H} \int_{0}^{h_{v}} \rho_{v} U_{v} d y$

The above expression allows for the determination of the pressure gradient, $d p / d x$, as a function of the imposed mass flux, $G$, the densities, $\rho_{l}$ and $\rho_{v}$, the viscosities, $\mu_{l}$ and $\mu_{v}$, and the liquid layer thickness $h_{l}$. The liquid layer thickness, $h_{l}$, is based on the void fraction, $\alpha$, that is determined from the Barcozy correlation (Eq. 16) and two other independent methods described in sections 5.3 and 5.4. The flow quality, $x$, needed to compute the void fraction is an experimental paremeter described by Eq. (1) in section 3.3. The pressure gradient obtained in this manner enables the specification of the velocity profile in each phase by Eq. (9). This velocity profile describes the fullydeveloped flow for the given experimental condition.

The dimensionless governing equations for adiabatic, incompressible flow in the upper vapor layer are:

$\nabla \cdot u_{v}=0, \quad \frac{\partial u_{v}}{\partial t}+\left(u_{v} \cdot \nabla\right) u_{v}=\frac{1}{R e} \Delta u_{v}-\nabla p-\frac{1}{F r^{2}} \hat{\mathbf{e}}_{y}$

In the lower liquid layer, the equations read:

$\nabla \cdot u_{l}=0, \quad \frac{\partial u_{l}}{\partial t}+\left(u_{v} \cdot \nabla\right) u_{v}=\frac{m}{r R e} \Delta u_{l}-\frac{1}{r} \nabla p-\frac{1}{F r^{2}} \hat{\mathbf{e}}_{y}$

where $u_{v}$ and $u_{l}$ are the dimensionless velocities in the vapor and liquid layers, respectively, $p$ denotes the pressure and $\hat{\mathbf{e}}_{y}$ is a unit vector in the $y$-direction.

The above equations are made dimensionless by scaling the velocity with $U_{0}$, length with $h_{v}$, time with $h_{v} / U_{o}$, and pressure with $\rho_{v} U_{0}^{2}$. The dimensionless parameters can be expressed as:

$R e=\frac{\rho_{v} h_{v} U_{o}}{\mu_{v}}, \quad W e=\frac{\rho_{v} h_{v} U_{o}^{2}}{\gamma}, \quad F r=\frac{U_{o}}{\sqrt{g h_{v}}}$

where $U_{0}$ is the velocity at $y=h_{v}$, i.e. the maximum velocity in the channel, $R e$ is the Reynolds number, We the Weber number, and $\mathrm{Fr}$ the Froude number. The ratios of viscosity, density, and layer thickness are denoted, respectively, by:

$m=\frac{\mu_{l}}{\mu_{v}}, \quad r=\frac{\rho_{l}}{\rho_{v}}, \quad n=\frac{h_{v}}{h_{l}}$

The vapor and liquid layer thicknesses, $h_{v}$ and $h_{l}$, respectively, are determined from the Baroczy void fraction correlation and two other independent methods described in sections 5.3 and 5.4. The Baroczy correlation for void fraction, $\alpha$, given in Eq. (16) (Baroczy, 1965), is based on the turbulent Martinelli parameter and is generally 
considered to be applicable over a range of geometric and flow conditions with an expected standard deviation of $\pm 20 \%$.

$\alpha=\left[1+r^{-0.65} m^{0.13}\left(\frac{1-x}{x}\right)^{0.74}\right]^{-1}$

Where $x$ is the thermodynamic quality given by equation (1). The dimensionless film thickness is then:

$n=\frac{h_{v}}{h_{l}}=\frac{\alpha}{1-\alpha}$

In view of the fact that the flow could be turbulent in the vapor layer, the hydrodynamic vapor mass fraction associated with the fully developed basic flow, based on $\alpha$ defined by Eq. (16), may differ somewhat from the thermodynamic quality $x$ defined in Eq. (1). In sections 5.3 and 5.4 we consider two alternatives methods for specifying $\alpha$ that are based on matching vapor mass fraction with thermodynamic flow quality and the observed wavelengths with linear stability results, respectively, and compare with results based on Eq. (16).

To quantify the behavior of small amplitude perturbations, a linear stability analysis is carried out. The appendix contains a detailed description of the procedure. The linear stability problem can be expressed as:

$c=c(k, R e, W e, F r, m, n, r)$

where $c=c_{r}+\mathrm{i} c_{i}$ is the complex growth rate obtained by solving the eigenvalue problem described in the Appendix. When $c_{i}>0$, the disturbance grows exponentially with time until the onset of nonlinearity. If $c_{i}<0$, the flow is said to be stable and $c_{i}=0$ indicates the state of neutral stability. The Chebyshev collocation spectral method is used to solve the eigenvalue problem for the eigenvalue, $c$, as described in the Appendix. This approach is well suited for non-periodic domains and also allows the solution to converge at an exponential rate. It has been used effectively in the past for similar problems (Kaffel and Renardy, 2010; Kaffel and Renardy, 2011; Kaffel and Riaz, 2015). For a more detailed description of the method, see Trefethen (2000), Boyd (2000) and Peyret (2002).

\subsection{Influence of Flow Quality and Mass Flux on Interfacial Wave Growth Rate}

Results obtained from the linear stability analysis are described in terms of flow parameters, $R e, W e, F r, r, m$ and $n$ that were chosen to match specific experimental conditions. The mass flux, $G$, flow quality, $x$, and other, dimensionless flow parameters are listed in Table 6 for each experimental condition. The viscosity ratio, $m$, varies between 29 and 33, the density ratio, $r$, between 85 and 113, the flow quality, $x$, between $38 \%$ and $87 \%$, and mass flux, $G$, between 220 and $620 \mathrm{~kg} / \mathrm{m}^{2}-\mathrm{s}$. 
Figure 8 plots the results of linear stability analysis computed with the method described in section 5.1 and the Appendix. The basic state is computed using the void fraction given by Eq. (16). Linear stability results are presented in the form of growth rate versus wavenumber curves. The dimensionless wave growth rate, $\sigma$, is defined by $\sigma=k c_{i}$, where $k$ is the wavenumber in dimensionless form and $c_{i}$ is the imaginary part of the wave speed (see Appendix). Positive growth rates, i.e. values of $\sigma>0$ indicate the range of wavenumbers for which the flow is unstable while a negative growth rate, $\sigma<$ 0 , indicates stability. For wavenumbers larger than a threshold value, the growth rate is negative, implying that small wavelength features in the flow will be damped by viscous and surface tension effects. Within the unstable range of wavenumbers with $\sigma>0$, the growth rate increases from $\sigma=0$ at $k=0$ to a maximum value, $\sigma=\sigma_{m}$ at $k=k_{m}$, and then decays. The dominant wavenumber, $k_{m}$, related to the maximum growth rate, $\sigma_{m}$, is of interest because it is likely to be observed in physical systems perturbed with arbitrary disturbance patterns.

Stability curves shown in Fig. 8 are for a constant mass flux of $G=620 \mathrm{~kg} / \mathrm{m}^{2}$ $\mathrm{s}$, corresponding to the experimental cases presented in section 4.1.3. For these cases the dimensionless layer thickness $n=14,22$ and 48 for a quality of $x=54 \%, 70 \%$ and $87 \%$, respectively. Figure 8 shows that with an increase in flow quality, $x$, the maximum growth rate, $\sigma_{m}$, decreases and the maximum wavenumber, $k_{m}$, increases slightly. The decrease in $\sigma_{m}$ implies that the relative stability of the liquid film increases with increasing flow quality. These results appear to be in qualitative agreement with experimental observations presented in section 4.1.3, which indicate that the interfacial wave patterns become more ordered with an increase in the flow quality and that the size of the average wave decreases. A more detailed quantitative comparison is presented in the next section.

The dimensionless numbers in Table 6 indicate that for a fixed mass flow rate, $R e$ and $W e$ are almost independent of $x$. This can be understood by considering that $R e$ and $W e$ are based on the maximum velocity of the vapor phase, $U_{0}$, which does not change appreciably with changes in $x$ because of the relatively small corresponding changes in the void fraction, i.e., $\alpha=0.93,0.96$ and 0.98 , for the cases considered in Fig. 8 . Moreover, these large values of $\alpha$ indicate that the flow occurs primarily through the vapor phase. Hence, the maximum velocity in the vapor layer does not vary substantially with small changes in the void fraction. Consequently, $R e$ and $W e$ are not very sensitive to changes in thermodynamic quality for a fixed mass flux in all cases. Moreover, all experimental cases are also characterized by very small values of the Bond or the Eotvos number, $E o=\Delta \rho g h_{v}^{2} / \gamma<0.01$, and are therefore not expected to be influenced appreciably by changes in density.

Table 6 lists additional dimensional parameters based on the height of the liquid layer, $h_{l}$, the viscosity and density of the liquid phase, $\mu_{l}$, and $\rho_{l}$, respectively, and the interfacial velocity, $U_{v}(0)=U_{l}(0)=U_{I}$. The corresponding dimensionless numbers, $R e_{I}$ and $W e_{I}$, decrease steadily with an increase in the thermodynamic quality, $x$, which correlates well with the smaller growth rates for higher qualities, and thinner liquid films, as observed in Fig. 8. The stability characteristics for such cases thus appear to be correlated more with the flow behavior in the vicinity of the interface, indicating a dominance of the interfacial mode of instability. Kaffel and Riaz (2015) have recently investigated this mode of instability in more detail. The shear mode of instability, on the 
other hand, is expected to play a bigger role for larger values of $U_{0}$, corresponding to values of $G$ greater than those considered in this study.

The influence of mass flux, $G$, is considered in Fig. 9, for $x=78 \%, 72 \%$ and $70 \%$ for $G=220 \mathrm{~kg} / \mathrm{m}^{2}-\mathrm{s}, G=420 \mathrm{~kg} / \mathrm{m}^{2}-\mathrm{s}$ and $G=620 \mathrm{~kg} / \mathrm{m}^{2}$-s, respectively, with $\delta=184 \mu \mathrm{m}, m=33, n=20, r=100$ and $E o<0.01$. The objective is to attempt to understand the influence of mass flux on the stability behavior while holding the flow quality, $x$, and layer thickness ratio, $n$, approximately constant. Figure 9 shows that the maximum growth rate, $\sigma_{m}$, and the maximum wavenumber, $k_{m}$, both increase with an increase in mass flux, $G$. The corresponding values of the dimensionless numbers noted in Table 6 indicate that an increase in $\sigma_{m}$ is associated with a corresponding increase in both the Reynolds number and the Weber number, from $R e=2746$ and $W e=50$ at $G=220 \mathrm{~kg} / \mathrm{m}^{2}$-s, to $R e=7510$ and $W e=337$ at $G=620 \mathrm{~kg} / \mathrm{m}^{2}$-s. Other parameters, $W e, R e_{l}$, and $W e_{l}$, follow a similar trend. For these cases, both sets of dimensionless numbers, i.e., those related to velocity scales $U_{0}$ and $U_{I}$, are clearly positively correlated with the maximum growth rate, $\sigma_{m}$ because both $U_{0}$ and $U_{I}$ increase proportionately with an increase in $G$ for a relatively fixed value of $\alpha$. However, while the maximum growth rate increases uniformly with the mass flux, $G$, both $k_{m}$ and the range of the unstable spectrum for which $\sigma>0$ appear to reach a limiting value.

\subsection{Experimental and Numerical Wavelength Comparison}

Linear stability results can be compared with experimental observations on the basis of the wavelength of interfacial patterns observed at the interface. The average wavelength obtained from the linear stability analysis can be expressed in dimensional form as

$\bar{\lambda}=\frac{2 \pi}{k_{m}} \bar{h}_{v}$.

where $k_{m}$ is the most unstable wavenumber related to the maximum growth rate, $\sigma_{m}$, and $\bar{h}_{v}$ is the average vapor layer thickness.

In order to compare with the linear stability results, the range of wavelengths for interfacial waves is experimentally measured by directly illuminating the upper film with a flash. The illumination produces shadows that coincide with the wave crests, an example of which is shown in Fig. 10(a) for the $x=43 \%$ and $G=420 \mathrm{~kg} / \mathrm{m}^{2}$-s case. An image processing software, Photoshop in this case, is then used to convert the wave shadows into a 2-bit image, as shown in Fig. 10(b). Matlab is subsequently used to measure the streamwise wavelengths of the 2-bit image to produce the wavelength distribution shown in Fig. 10(c). Note that the wavelengths are only measured in the second half of the channel (the last $17.5 \mathrm{~mm}$ ) in order to mitigate the influence of entry effects

In view of the fact that the basic velocity profile is based on the void fraction determined from the Baroczy correlation for turbulent flow, we expect the associated vapor mass fraction to deviate somewhat from the thermodynamic quality, $x$, defined by Eq. (1). This discrepancy can potentially introduce uncertainty in the comparison of linear stability results with experimental observations. To be able to better understand 
this aspect, we calculate the difference between the thermodynamic quality, $x$, defined in Eq. (1) and the hydrodynamic vapor mass fraction, $x_{\alpha}$, that is associated with the fully developed flow, for which the void fraction, $\alpha$, is given by the Baroczy correlation defined in Eq. (16). The hydrodynamic vapor mass fraction, $x_{\alpha}$, associated with the fully developed basic state is computed according to:

$x_{\alpha}=\frac{\rho_{v}}{G H} \int_{0}^{H \alpha} U_{v, \alpha} d y$

where the subscript $\alpha$ indicates that the velocity profile, $U_{v, \alpha}$, is based on the void fraction given by Eq. (16). The hydrodynamic quality, $x_{\alpha}$, can be compared with the experimental thermodynamic quality, $x$, as the difference, $\left|x-x_{\alpha}\right| / x$, which is tabulated in Table 7. The difference is relatively large for smaller values of $x$, but decreases systematically as the quality increases for a fixed value of $G$. This indicates that the void fraction, $\alpha$, given by the Baroczy correlation is relatively more consistent for larger values of $x$.

The consideration of the difference, $\left|x-x_{\alpha}\right|$, suggests another approach for determining a void fraction for which $\left|x-x_{\alpha}\right|=0$, for the same mass flux. Towards that end, we define a fully developed basic state, $U_{v, \widetilde{\alpha}}$, that is based on an adjusted void fraction, $\tilde{\alpha}$, which is obtained by matching the vapor mass fraction:

$x_{\widetilde{\alpha}}=\frac{\rho_{v}}{G H} \int_{0}^{H \widetilde{\alpha}} U_{v, \widetilde{\alpha}} d y$,

with the thermodynamic quality, $x$ (Eq. 1). The basic state, $U_{v, \widetilde{\alpha}}$, is based on the adjusted void fraction, $\tilde{\alpha}$, which corresponds to the condition, $\left|x-x_{\widetilde{\alpha}}\right| / x<10^{-2}$. This condition is satisfied by gradually varying the void fraction until the desired accuracy is achieved.

The difference, $|\alpha-\tilde{\alpha}| / \alpha$, between the adjusted void fraction, $\tilde{\alpha}$, and the void fraction based on the Baroczy correlation, $\alpha$, is listed in Table 7. Interestingly, this discrepancy is more consistent with the accuracy of the Baroczy correlation. Moreover, the difference in void fractions, $|\alpha-\tilde{\alpha}| / \alpha$, is always less than the difference in flow qualities, $\left|x-x_{\alpha}\right| / x$, more so for smaller values of $G$ and $x$. The dimensional parameters corresponding to the basic state computed on the basis of the adjusted void fraction, $\tilde{\alpha}$, are also listed in Table 7. The relative difference (not listed) between these and the ones given in Table 6 , which are associated with the void fraction, $\alpha$, is greater than $\left|x-x_{\alpha}\right| / x$ but less then $|\alpha-\tilde{\alpha}| / \alpha$. To find the influence of such changes on the stability behavior, we determined two sets of linear stability results with the basic state computed using the void fractions $\alpha$ and $\tilde{\alpha}$.

The experimental distribution of measured wavelengths is compared with the linear stability results in Figs. 11-13 for the mass flux of 220,420 , and $620 \mathrm{~kg} / \mathrm{m}^{2}-\mathrm{s}$, respectively. Two sets of data are plotted for the linear stability analysis. The first set, shown with dashed lines, is based on the dimensionless parameters listed in Table 6, corresponding to the void fraction, $\alpha$, while the second set, shown in symbols, is based on dimensionless parameters in Table 7, corresponding to the void fraction, $\tilde{\alpha}$. Experimental 
data (solid lines) is presented in Figs. 11-13 as a probability distribution of measured wavelengths, normalized by the maximum probability. The linear stability results for growth rate vs. wavenumber curves are normalized by the maximum growth rate. In this comparison, the growth rate distribution is interpreted as a predicted probability distribution of experimentally observed interfacial wavelengths.

Despite the complex nature of flow in the microchannel, Figs. 11-13 show that the distribution of wavelengths predicted by the linear stability analysis agrees reasonably well with the range of wavelengths observed in the experiments, most notably around the most unstable wavelength. The two sets of linear stability results based on $\alpha$ and $\tilde{\alpha}$ are observed to be quite similar over much of the wavenumber range considered. The most probable experimental wavelength and the most unstable theoretical wavelength based on the void fraction $\tilde{\alpha}$ are tabulated and compared in Table 8 . The agreement appears to be quite good with a maximum absolute difference of less then $10 \%$. The lack of full agreement can be attributed to features not fully accounted for by the linear stability analysis such as the spanwise wave structure, the turbulent nature of flow in the vapor layer and the saturated nonlinear state of interfacial waves. On the other hand, the relative agreement indicates that the use of a laminar basic state for carrying out the linear stability analysis allow for an effective prediction of experimental wavelengths. Moreover, it further implies that the dominant wavelengths predicted by the linear results persist in the nonlinear regime with apparently little wavelength coarsening. Such behavior in parallel flows with time invariant basic states is different compared to problems involving time decaying basic states (Tilton et al., 2013; Daniel et al., 2015) where substantial wavelength coarsening occurs before the transition to the nonlinear regime.

\subsection{Discussion.}

The basic state employed by the linear stability analysis relies on the calculation of the film thickness or height, which is based on the void fraction that is obtained from the Barcozy correlation, expressed by Eq, 16. The Barcozy correlation is expected to be accurate to within a standard deviation of $\pm 20 \%$. The comparison of stability results with experimental data reported in Figs. 11-13 using two different methods for computing the layer thickness indicates that the deviation is relatively mild. In order to further evaluate the validity of the Barcozy correlation, the linear stability analysis is used to carry out an additional inverse calculation of film thickness. The procedure involves systematically varying the liquid film thickness, $h_{l}$, until the dominant wavelength predicted by the linear stability analysis matches exactly the dominant wavelength observed in the corresponding experiment. Figure 14 plots the liquid film thickness, $h_{l}$, obtained with the inverse calculation as a function of the dominant wavenumber, $k_{m}$, for various experimental conditions noted in Table 6 . The values of $h_{l}$ based on the Baroczy correlation for corresponding experimental conditions are shown with symbols. The maximum and minimum values of $h_{l}$ indicate the uncertainty in the measurement of the mass flow rate. Figure 14 shows a reasonably good agreement between $h_{l}$ obtained from the linear stability analysis and the one given by the Barcozy correlation for the corresponding experimental conditions. This comparison thus further helps build 
confidence in the Barcozy correlation for the experimental conditions considered in this study.

Another source of uncertainty in the predictions of the linear stability analysis is related to the assumption of negligible gravitational effects. This assumption is motivated by the small thickness of the liquid layer compared with other channel dimensions. The practical advantage of this assumption is that symmetry can be assumed about the channel centerline leading to a simpler eigenvalue problem compared with the analysis of the full problem. In order to evaluate the validity of this approach we compare the results for the lower layer with those for the upper layer for $G=420 \mathrm{~kg} / \mathrm{m}^{2}-\mathrm{s}, r=105, m=$ $32, n=26$ and $E o=0.0085$. The latter calculation involves changing the direction of gravity (i.e., using a negative value for the gravitational constant, $g$ ). Figure 15 indicates that the two sets of results for the top and the bottom layers are in good agreement even for a relatively large density ratio of $r=105$. These results thus help support the assumption of negligible gravitational effect. This is understandable in view of a small value of $E o$ for the case considered in Fig. 14 as well as for all other experimental cases where Eo $<0.01$.

Finally, the basic state for velocity is taken to be the velocity profile associated with fully developed laminar flow. The large values of the Reynolds numbers for the vapor phase noted in Table 6, suggest that the flow can be expected to be turbulent within the vapor layer. In the liquid layer, however, the Reynolds numbers are substantially lower and a fully developed flow is expected. Moreover, a recent study by Kaffel and Riaz (2015) indicates that the eigenvectors of the unstable interfacial modes are generally localized in the neighborhood of the interface where the local Reynolds numbers are sufficiently low when the film thickness is small. Hence, close to the interface, the fully developed velocity profile may represent a reasonable approximation in both layers. The effect of turbulent eddies in the vapor layer on wave patterns at the interface and the associated implications for liquid layer receptivity (Saric et al., 2002), however, have not been accounted for in the current approach. Despite such constraints, a reasonable agreement between the linear stability results and experimental observations lends some confidence to the use of the fully developed velocity profile as the basic state for the cases considered in this study.

\section{Conclusions}

In the present study, wave patterns generated on the surface of the liquid layer in two-phase annular flow of FC-72 in a microgap channel are photographically visualized and compared to predictions obtained from linear stability theory. The deformations and emerging patterns observed at the liquid vapor interface are of particular interest and a summary of the significant finding is as follows:

- Within annular flow, periodic waves are consistently observed at the liquid film interface, traveling along the streamwise length of the channel. At a mass flux of $220 \mathrm{~kg} / \mathrm{m}^{2}$-s, the interfacial waves have crests that are 3-D in shape, smooth, and widely spaced. With increasing flow quality, both the wavelength and amplitude of the 3-D waves decrease but the general 3-D wave pattern persists. As the mass flux increases from 220 to $420 \mathrm{~kg} / \mathrm{m}^{2}-\mathrm{s}$, the interfacial waves become "scaly" in 
appearance, unsteady, and tightly spaced; this pattern persists at a mass flux of $620 \mathrm{~kg} / \mathrm{m}^{2}$-s. Regardless of mass flux, small amplitude, tightly spaced waves with continuous spanwise fronts appear at large flow qualities.

- In an effort to predict the most probable wavelength at the liquid-vapor interface, a linear stability analysis of immiscible annular channel flow is carried out for all experimental cases studied. The theoretically predicted wavelengths are found to generally agree with experimental results. This suggests that the current approach is useful for characterizing interfacial waves in shear-driven liquid flow in channels, albeit for small Eotvos numbers. This aspect would be important for the analysis and modeling of diabatic flows because the spatial development of thermal fluctuations is expected to be correlated with the structure of hydrodynamic waves.

\section{Acknowledgement}

This study was supported by the Office of Naval Research, Grant No. N00014-111-0468 monitored by Dr. Mark Spector.

\section{References}

Alam, T., Lee, P.S., Yap, C.R., Jin L., 2012. Experimental investigation of local flow boiling heat transfer and pressure drop characteristics in microgap channel, Int. Journal of Multiphase Flow 42, 164-174.

Bar-Cohen, A., Albrecht, J.D., Maurer, J.J., 2011. Near-Junction Thermal Management for Wide Bandgap Devices. Compound Semiconductor Integrated Circuit Symposium, 1-5.

Bar-Cohen, A., Geisler, K.J.L., 2011. Cooling the Electronic Brain. Mechanical Engineering Magazine, April.

Bar-Cohen, A., Rahim, E., 2009. Modeling and prediction of two-phase microgap channel heat transfer characteristics, Heat Transfer Eng. 30(8), 601-625.

Bar-Cohen, A., Rahim, E., Ali, I.A., 2012. Two-Phase Microgap Cooling of a ThermallySimulated Microprocessor Chip. Thirteenth Intersociety Conference on Thermal and Thermomechanical Phenomena in Electronic Systems, San Diego, USA.

Bar-Cohen, A., Sheehan, J.R., Rahim, E, 2012. Two-Phase Thermal Transport in Microgap Channels-Theory, Experimental Results, and Predictive Relations. Microgavity Sci. Tech. 24, 1-15.

Baroczy, C.J., 1965. Correlation of liquid fraction in two-phase flow with applications to liquid metals, Chem. Eng. Prog. Symp. 61, 179-191.

Boyd, J. P., 2000. Chebyshev and Fourier Spectral Methods. DOVER Publications, New York.

Cortina-Diaz, M., Schmidt, J., 2006. Flow boiling heat transfer of n-hexane and n-octane in a minichannel. Proceedings of the 13th International Heat Transfer Conference, Sydney, Australia. 
Daniel, D., Riaz, A., Tchelepi, H.A., 2015. Onset of Natural Convection in Layered Aquifers. J. Fluid Mech. 767, 763-781.

Drazin, P.G., 2001. Introduction to Hydrodynamic Stability. Cambridge University Press, Cambridge.

Drazin, P.G., Reid, W.H., 1981. Hydrodynamic Stability. Cambridge University Press, Cambridge.

Hooper, A., Boyd, W., 1987. Shear-flow instability due to a wall and a viscosity discontinuity at the interface. J. Fluid Mech. 179, 201-225.

Houshmand, F., Peles, Y., 2013.Convective heat transfer to shear-driven liquid film flow in a microchannel. Int. J. Heat Mass Trans. 64, 42-52.

Kabov, O.A., Zaitsev, D.V., Cheverda, V.V., Bar-Cohen, A., 2011. Evaporation and flow dynamics of thin, shear-driven liquid films in microgap channels. Exp. Therm. Fluid Sci. 35, 825-831.

Kaffel, A., Renardy, M., 2010. On the stability of plane parallel viscoelastic shear flows in the limit of infinite Weissenberg and Reynolds numbers. J. Non-Newt. Fluid Mech. 165(23-24), 1670-1676.

Kaffel, A., Renardy, M., 2011. Surface modes in inviscid free surface shear flows. J. App. Math. Mech. 91(8), 649-652.

Kaffel, A., Riaz, A., 2015. Eigenspectra and mode coalescence of temporal instability in twophase channel flow. Phys. Fluids 27, 042101.

Kim, D., Rahim, E., Bar-Cohen, A., Han, B., 2010. Direct Submount Cooling of High-Power LEDs. IEEE Trans. Comp. 33, 698-712.

Kim, D.W., Rahim, E., Bar-Cohen, A., Han, B., 2008. Thermofluid Characteristics of Two-Phase Flow in Micro-Gap Channels. 11th Intersociety Conference on Thermal and Thermomechanical Phenomena in Electronic Systems, San Diego, 979-992.

Kopp, B.A., Oullette, E., Billups, A.J., 2000. Thermal design considerations for wide bandgap transistors. Microwave J. 43, 110-118.

Mastrullo, R., Mauro, A.W., Thome, J.R., Vanoli, G.P., 2012. CO2 and R410A: Two-phase flow visualizations and flow boiling measurements at medium (0.50) reduced pressure. App. Therm. Eng. 49, 2-8.

Peyret, R., 2002. Spectral Methods for Incompressible Viscous Flow, Vol. 148 of Appl. Math. Sci., Springer, New York.

Rahim, E., Revellin, R., Thome, J., Bar-Cohen, A., 2011. Characterization and prediction of twophase flow regimes in miniature tubes. Int. J. Multiphase Flow 37, 12-23.

Saric, W.S., Reed, H.L., Kerschen, E.J., 2002. Boundary-layer receptivity to freestream disturbances. Ann. Rev. Fluid Mech. 34(1), 291-319.

Thome, J.R., Bar-Cohen, A., Revellin, R., Zun, I., 2013. Unified Mechanistic Multiscale Mapping of Two-Phase Flow Patterns in Microchannels. Exp. Therm. Fluid Sci. 44, 1-22.

Tilton, N., Daniel, D., Riaz, A., 2013. The initial transient period of gravitationally unstable diffusive boundary layers developing in porous media. Phy. Fluids 25, 092107. 
Trefethen, L. N., 2000. Spectral Methods in Matlab. SIAM, Philadelphia PA.

Yang, Y., Fujita, Y., 2004. Flow boiling heat transfer and flow pattern in rectangular channel of mini-gap. Second International Conference on Microchannels and Minichannels (ICMM2004-2383), New York, USA.

Yen, T.H., Kasagi, N., Suzuki, Y., 2003. Forced convective boiling heat transfer in microtubes at low mass and heat fluxes. Int. J. Multiphase Flow 29, 1771-1792.

Yih, C.-S., 1967. Instability due to viscosity stratification. J. Fluid Mech. 27, 337-352.

\section{Appendix}

To carry out a linear perturbation analysis, the dimensionless velocity, pressure, and the interface location are expanded in terms of the base state and a perturbation:

$$
\begin{aligned}
& u_{v}=\hat{u}_{v}+u_{v}^{\prime} \\
& u_{l}=\hat{u}_{l}+u_{l}^{\prime} \\
& v_{v}=v_{v}^{\prime} \\
& v_{l}=v_{l}^{\prime} \\
& p=P+p^{\prime}
\end{aligned}
$$

where $\hat{u}_{v}$ and $\hat{u}_{l}$ denote the velocity base states defined below in Eqs. (A23) and (A24). The velocity perturbations are expressed in terms of the stream functions, $\psi_{v}$ and $\psi_{l}$ :

$u_{v}^{\prime}=\frac{\partial \psi_{v}}{\partial y}$

$u_{l}^{\prime}=\frac{\partial \psi_{l}}{\partial y}$

$v_{v}^{\prime}=-\frac{\partial \psi_{v}}{\partial x}$

$v_{l}^{\prime}=-\frac{\partial \psi_{l}}{\partial x}$

The linearized equations and boundary conditions suggest a normal mode analysis:

$\left(\psi_{v}, \psi_{l}, p^{\prime}, \eta\right)=\left(\varphi_{v}, \varphi_{l}, f, h\right) \exp [\mathrm{i} k(x-c t)]$ 
where $\eta$ denotes the $y$-coordinate of the interface, $\varphi_{v}, \varphi_{l}, f, h$ are the disturbance amplitudes, $k=2 \pi h_{v} / \lambda$ is the dimensionless wavenumber related to the wavelength, $\lambda$, $c=c_{r}+\mathrm{i} c_{i}$ is the complex wave velocity, and $\sigma=k c_{i}$ is the growth rate.

Upon substitution of the expressions (A1) - (A9) into the equations of motion (12) - (13), and by neglecting quadratic terms and using the resulting linearized equations in Eq. (A10), we obtain the familiar Orr-Sommerfeld equations for each phase:

$$
\begin{aligned}
& \varphi_{v}^{\mathrm{IV}}-2 k^{2} \varphi_{v}^{\prime \prime}+k^{4} \varphi_{v}=\mathrm{i} k \operatorname{Re}\left[\left(U_{v}-c\right)\left(\varphi_{v}^{\prime \prime}-k^{2} \varphi_{v}\right)-U_{v}^{\prime \prime} \varphi_{v}\right] \\
& \varphi_{l}^{\mathrm{IV}}-2 k^{2} \varphi_{l}^{\prime \prime}+k^{4} \varphi_{l}=i \frac{k r}{m} \operatorname{Re}\left[\left(U_{l}-c\right)\left(\varphi_{l}^{\prime \prime}-k^{2} \varphi_{l}\right)-U_{l}^{\prime \prime} \varphi_{l}\right]
\end{aligned}
$$

These equations will be solved subject to eight boundary conditions, two at the solid boundary, two at the free surface, and four at the interface. At the wall and free surface boundaries, $y=-1 / n$ and $y=0$, respectively, the no slip condition is used. Thus we have:

$$
\begin{aligned}
& \varphi_{v}(1)=0 \\
& \varphi_{v}^{\prime}(1)=0 \\
& \varphi_{l}(-1 / n)=0 \\
& \varphi_{l}^{\prime}(-1 / n)=0
\end{aligned}
$$

At the interface the velocity is continuous, the jump in normal stress is balanced by surface tension and the kinematic free surface condition holds. The linearized boundary conditions at $y=0$ satisfy:

$$
\begin{aligned}
& \varphi_{l}(0)=\varphi_{v}(0) \\
& \varphi_{l}^{\prime}(0)-\varphi_{v}^{\prime}(0)=\left(\alpha_{2}-\alpha_{1}\right) \varphi_{l}(0) / c_{0} \\
& \varphi_{v}^{\prime \prime}(0)+k^{2} \varphi_{v}(0)=m\left(\varphi_{l}^{\prime \prime}(0)+k^{2} \varphi_{l}(0)\right)+2\left(m \beta_{2}-\beta_{1}\right) \varphi_{l}(0) / c_{0} \\
& -\mathrm{i} k \operatorname{Re}\left[c_{0} \varphi_{v}^{\prime}(0)+\alpha_{1} \varphi_{v}(0)\right]-\varphi_{v}^{\prime \prime \prime}(0)+3 k^{2} \varphi_{v}^{\prime}(0) \\
& +\mathrm{i} k r \operatorname{Re}\left[c_{0} \varphi_{l}^{\prime}(0)+\alpha_{2} \varphi_{l}(0)\right]+m\left[\varphi_{l}^{\prime \prime \prime}(0)-3 k^{2} \varphi_{l}^{\prime}(0)\right] \\
& =\mathrm{i} k \operatorname{Re}\left(F+k^{2} / W e\right) \varphi_{l}(0) / c_{0}
\end{aligned}
$$

where

$$
\begin{aligned}
& c_{0}=c-U_{l}(0) \\
& F=(r-1) / F r^{2}
\end{aligned}
$$


Equations (A11) and (A12), along with boundary and interface conditions given by Eqs. (A13) to (A20), define an eigenvalue for the complex wave speed, $c=c_{r}+\mathrm{ic}_{\mathrm{i}}$. This eigenvalue problem is solved using a Chebyshev collocation spectral method (Trefethen, 2000; Boyd, 2000; Peyret, 2002).

The coefficients, $\alpha_{1}, \alpha_{2}, \beta_{1}, \beta_{2}$ in Eqs. (A18) - (A20) are related to the dimensionless basic flow velocity profile

$\hat{u}_{v}=\xi+\alpha_{1} \hat{y}+\beta_{1} \hat{y}^{2}$

$\hat{u}_{l}=\xi+\alpha_{2} \hat{y}+\beta_{2} \hat{y}^{2}$

where $\hat{u}_{v}=U_{v} / U_{0}$ and $\hat{u}_{l}=U_{l} / U_{0}$ are the dimensionless basic velocities for the vapor and liquid phase, respectively, and $\hat{y}=y / h_{v}$ represents the dimensionless $y$-coordinate. The dimensionless coefficients in Eqs. (A23) and (A24) are related to the dimensional coefficients, $c_{1}, c_{2}, c_{3}$ and $c_{4}$, in Eq. (9) according to

$\alpha_{1}=\frac{c_{1} h_{v}}{U_{0}}, \quad \alpha_{2}=\frac{c_{3} h_{v}}{U_{0}}, \quad \xi=\frac{c_{2}}{U_{0}}=\frac{c_{4}}{U_{0}}$

$\beta_{1}=\frac{d p / d x h_{v}^{2}}{2 \mu_{v} U_{0}}, \beta_{2}=\beta_{1} / m$

where

$$
\begin{aligned}
& c_{1}=-\frac{d p / d x h_{v}}{\mu_{v}}, \quad c_{2}=c_{4}=-\frac{d p / d x h_{l}}{2 \mu_{l}}\left(h_{l}+2 h_{v}\right), \quad c_{3}=c_{1} / m \\
& U_{0}=\frac{d p / d x h_{v}^{2}}{2 \mu_{v}}+c_{1} h_{v}+c_{2}
\end{aligned}
$$

Based on the relations in Eqs. (A27) and (A28) the dimensionless coefficients in Eqs. (A23) and (A24) can then be expressed as

$$
\begin{aligned}
& \alpha_{1}=\frac{2 m n^{2}}{m n^{2}+2 n+1}, \quad \alpha_{2}=\frac{2 n^{2}}{m n^{2}+2 n+1} \\
& \beta_{1}=-\frac{m n^{2}}{m n^{2}+2 n+1}, \quad \beta_{2}=-\frac{n^{2}}{m n^{2}+2 n+1}
\end{aligned}
$$




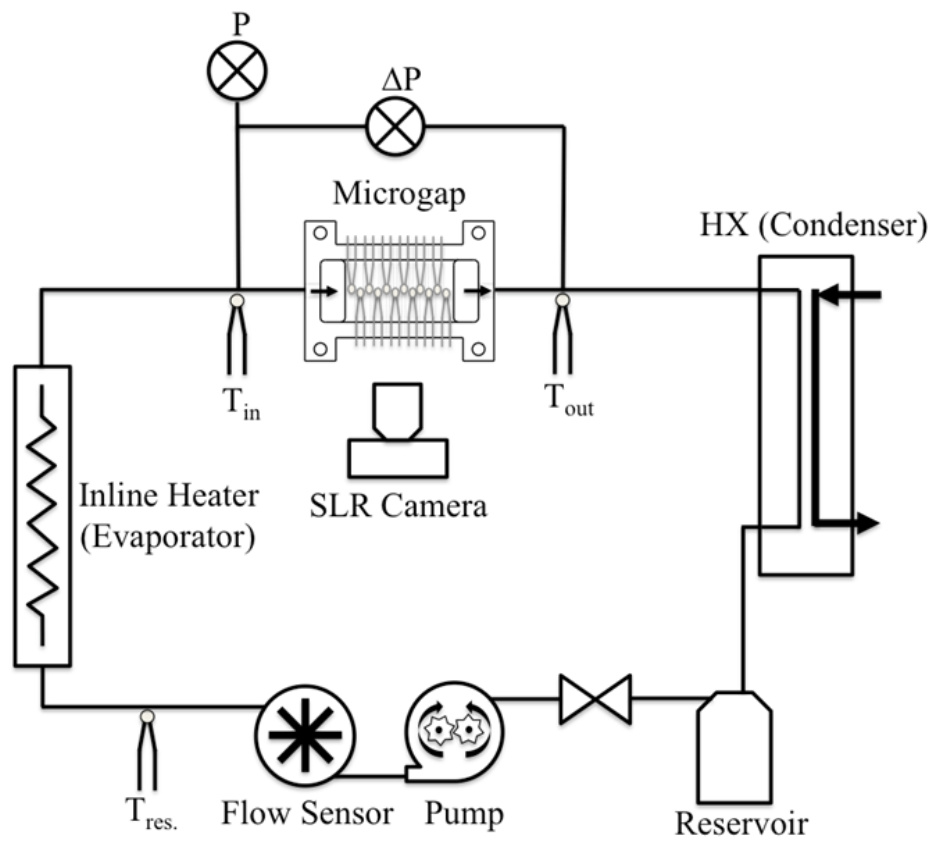

Fig. 1. Two-phase apparatus for present study.

(a)

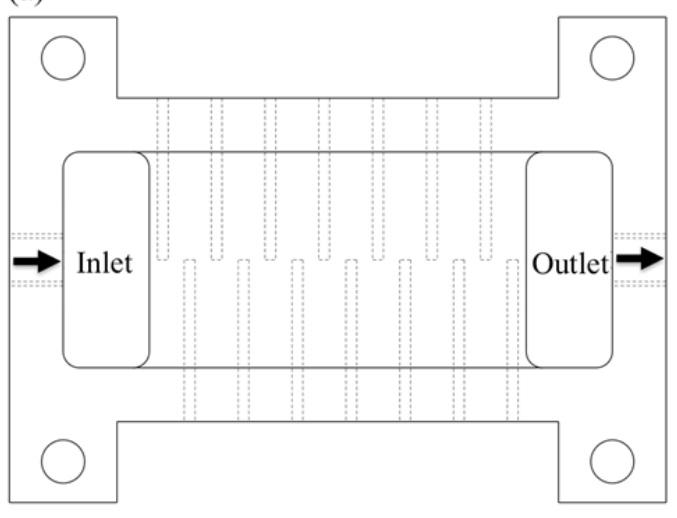

(b)

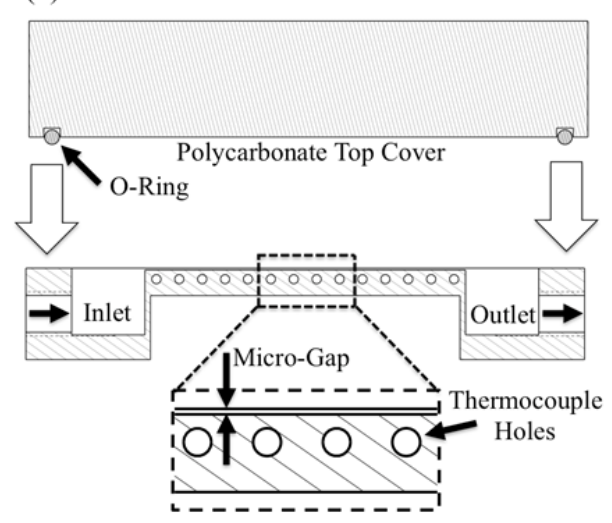

(c)

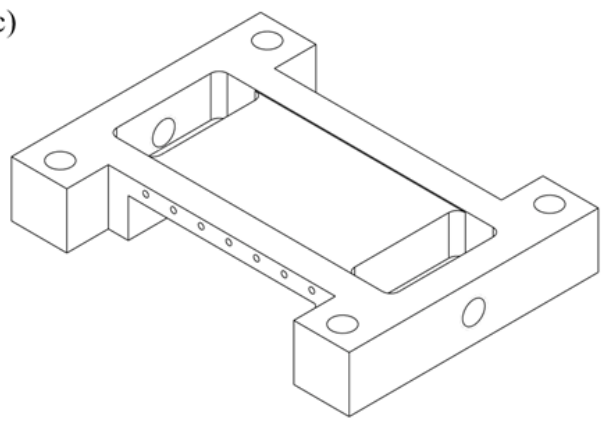

Fig. 2. (a) Top, (b) cross-sectional, and (c) isometric view of microgap test section. 
Table 1

Uncertainty in experimental parameters.

\begin{tabular}{lll}
\hline & Uncertainty & Uncertainty (\%) \\
\hline Temperature, $T(\mathrm{~K})$ & \pm 0.60 & $1.0-0.89 \%$ \\
Heat Input, $Q(\mathrm{~W})$ & $\pm 0.14-0.41$ & $0.23-0.47 \%$ \\
Flow Rate, $\dot{m}_{l}(\mathrm{~mL} / \mathrm{s})$ & \pm 0.03 & $2-6 \%$ \\
Channel Inlet Pressure, $P(\mathrm{kPa})$ & \pm 4.8 & $5.3-6.3 \%$ \\
Thermodynamic Quality, $x(\%)$ & $\pm 1.8-4.4$ & $2.6-6.6 \%$ \\
Superficial Liquid Velocity, $U_{s l}(\mathrm{~m} / \mathrm{s})$ & $\pm 0.0066-0.012$ & $5.1-26 \%$ \\
Superficial Vapor Velocity, $U_{s v}(\mathrm{~m} / \mathrm{s})$ & $\pm 0.68-1.5$ & $4.9-10 \%$ \\
Mass Flux, $G\left(\mathrm{~kg} / \mathrm{m}^{2}-\mathrm{s}\right)$ & $\pm 16-25$ & $3.7-6.7 \%$ \\
\hline
\end{tabular}

Table 2

Experimental test conditions and corresponding FC-72 properties.

\begin{tabular}{|c|c|c|c|c|c|c|c|c|c|c|c|}
\hline $\begin{array}{c}G \\
\mathrm{~kg} / \mathrm{m}^{2}-\mathrm{s}\end{array}$ & $\begin{array}{c}x \\
(\%)\end{array}$ & $\begin{array}{c}\Delta P \\
(\mathrm{kPa})\end{array}$ & $\begin{array}{c}T_{\text {sat,in }} \\
\left({ }^{\circ} \mathrm{C}\right)\end{array}$ & $\begin{array}{c}T_{\text {sat, out }} \\
\left({ }^{\circ} \mathrm{C}\right)\end{array}$ & $\begin{array}{c}T_{\text {sat,avg }} \\
\left({ }^{\circ} \mathrm{C}\right)\end{array}$ & $\begin{array}{l}P_{\text {avg }} \\
(\mathrm{kPa})\end{array}$ & $\begin{array}{c}\rho_{l}(\mathrm{~kg} / \\
\left.\mathrm{m}^{3}\right)\end{array}$ & $\begin{array}{c}\rho_{v} \\
\left(\mathrm{~kg} / \mathrm{m}^{3}\right)\end{array}$ & $\begin{array}{c}\mu_{l} \\
(\mathrm{~kg} / \mathrm{m}-\mathrm{s})\end{array}$ & $\begin{array}{c}\mu_{v} \\
(\mathrm{~kg} / \mathrm{m}-\mathrm{s})\end{array}$ & $\begin{array}{c}\gamma \\
(\mathrm{N}-\mathrm{m})\end{array}$ \\
\hline 220 & 39 & 8 & 58 & 57 & 57 & 105 & 1591 & 14 & $3.69 \mathrm{E}-04$ & $1.10 \mathrm{E}-05$ & $1.45 \mathrm{E}-02$ \\
\hline 220 & 54 & 10 & 59 & 57 & 58 & 106 & 1589 & 14 & $3.64 \mathrm{E}-04$ & $1.10 \mathrm{E}-05$ & $1.44 \mathrm{E}-02$ \\
\hline 220 & 66 & 10 & 59 & 58 & 58 & 106 & 1589 & 14 & $3.64 \mathrm{E}-04$ & $1.10 \mathrm{E}-05$ & $1.44 \mathrm{E}-02$ \\
\hline 220 & 78 & 13 & 60 & 58 & 59 & 108 & 1587 & 14 & $3.64 \mathrm{E}-04$ & $1.10 \mathrm{E}-05$ & $1.44 \mathrm{E}-02$ \\
\hline 420 & 43 & 20 & 62 & 57 & 59 & 112 & 1586 & 14 & $3.60 \mathrm{E}-04$ & $1.11 \mathrm{E}-05$ & $1.43 \mathrm{E}-02$ \\
\hline 420 & 56 & 27 & 63 & 57 & 60 & 115 & 1583 & 15 & $3.56 \mathrm{E}-04$ & $1.11 \mathrm{E}-05$ & $1.42 \mathrm{E}-02$ \\
\hline 420 & 72 & 30 & 64 & 57 & 61 & 116 & 1582 & 15 & $3.56 \mathrm{E}-04$ & $1.11 \mathrm{E}-05$ & $1.42 \mathrm{E}-02$ \\
\hline 420 & 85 & 33 & 65 & 57 & 61 & 118 & 1581 & 15 & $3.53 \mathrm{E}-04$ & $1.11 \mathrm{E}-05$ & $1.41 \mathrm{E}-02$ \\
\hline 620 & 38 & 44 & 68 & 57 & 62 & 123 & 1578 & 16 & $3.49 \mathrm{E}-04$ & $1.12 \mathrm{E}-05$ & $1.40 \mathrm{E}-02$ \\
\hline 620 & 54 & 60 & 71 & 57 & 64 & 131 & 1573 & 17 & $3.42 \mathrm{E}-04$ & $1.12 \mathrm{E}-05$ & $1.38 \mathrm{E}-02$ \\
\hline 620 & 70 & 76 & 74 & 57 & 66 & 139 & 1569 & 18 & $3.38 \mathrm{E}-04$ & $1.13 \mathrm{E}-05$ & $1.37 \mathrm{E}-02$ \\
\hline 620 & 87 & 90 & 77 & 58 & 67 & 146 & 1565 & 18 & $3.31 \mathrm{E}-04$ & $1.14 \mathrm{E}-05$ & $1.35 \mathrm{E}-02$ \\
\hline
\end{tabular}

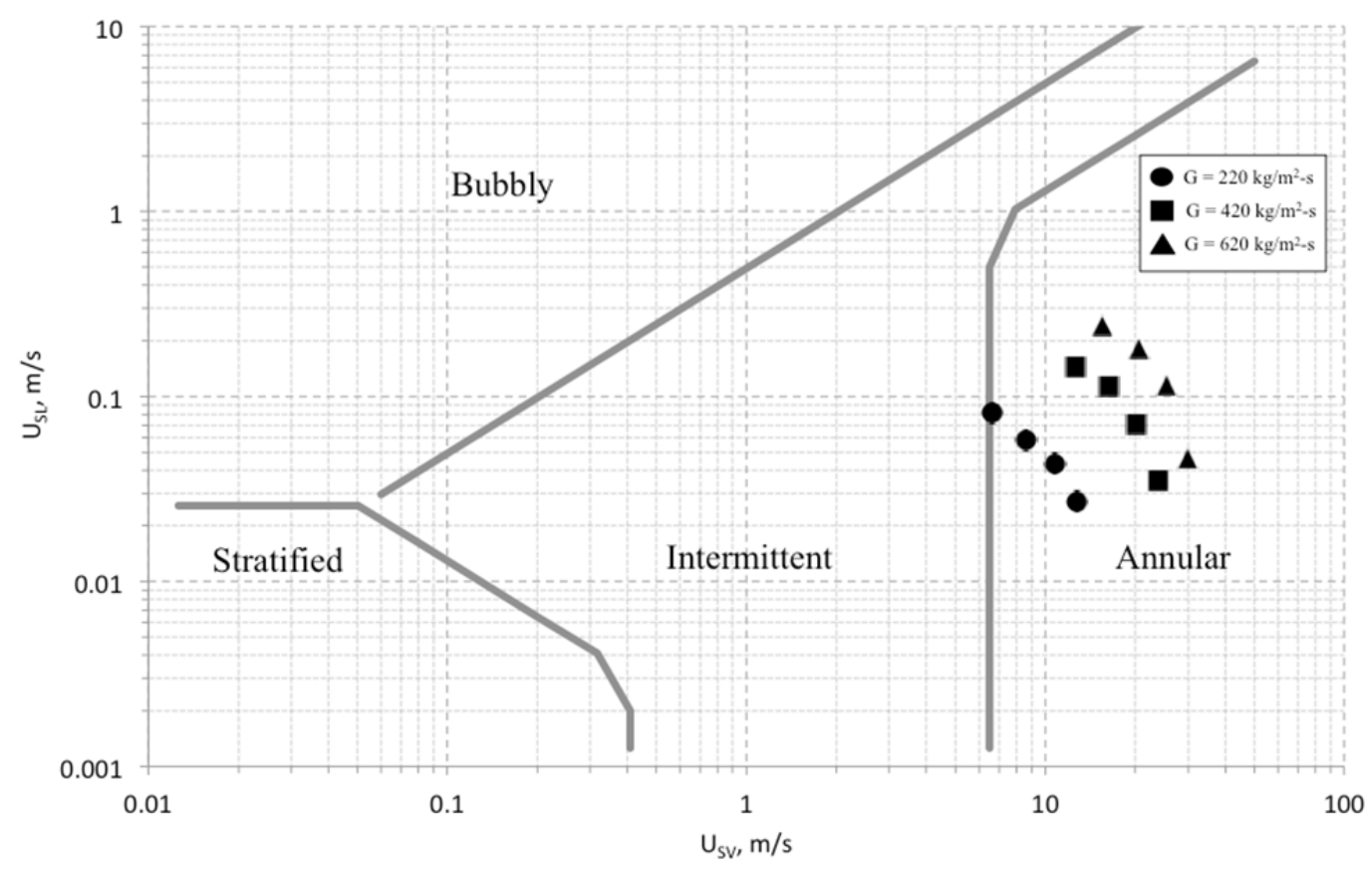


Fig. 3. Taitel-Dukler flow regime map with modified Ullman-Brauner bubbly-to-intermittent and intermittent-to-annular boundary for a $184 \mu \mathrm{m}$ microgap channel with FC-72 and loci of experimental cases.

(a) $U_{s v}=6.6 \mathrm{~m} / \mathrm{s}, U_{s l}=0.082 \mathrm{~m} / \mathrm{s}, x=39 \%$

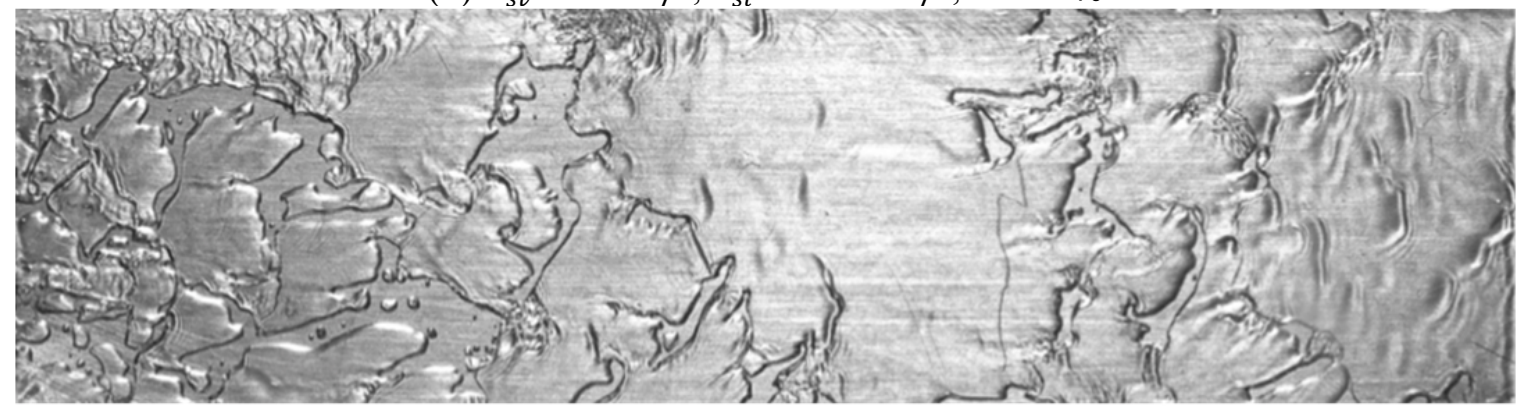

(b) $U_{s v}=8.6 \mathrm{~m} / \mathrm{s}, U_{s l}=0.058 \mathrm{~m} / \mathrm{s}, x=54 \%$

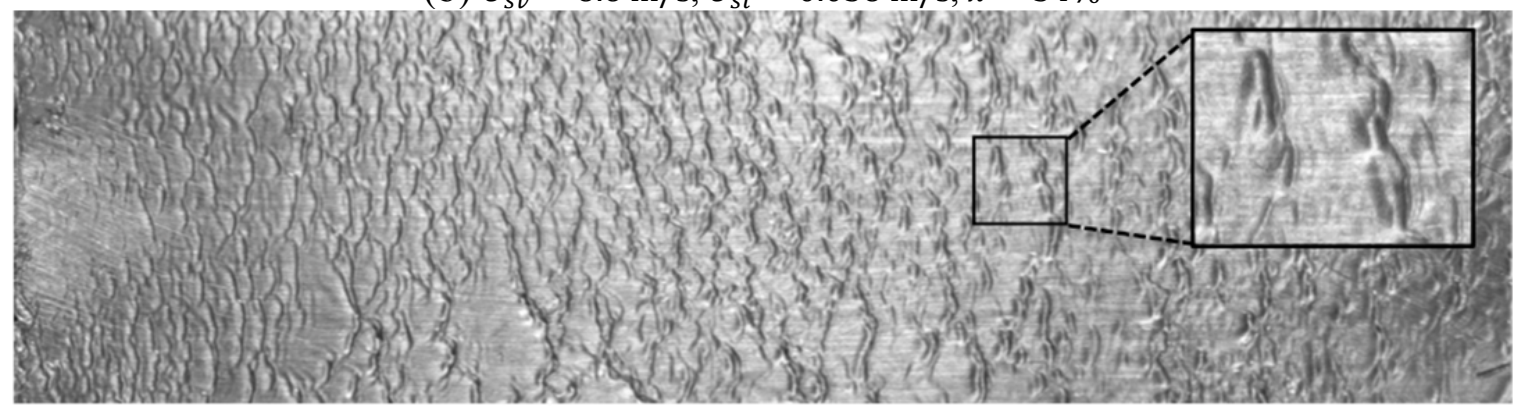

(c) $U_{s v}=11 \mathrm{~m} / \mathrm{s}, U_{s l}=0.043 \mathrm{~m} / \mathrm{s}, x=66 \%$

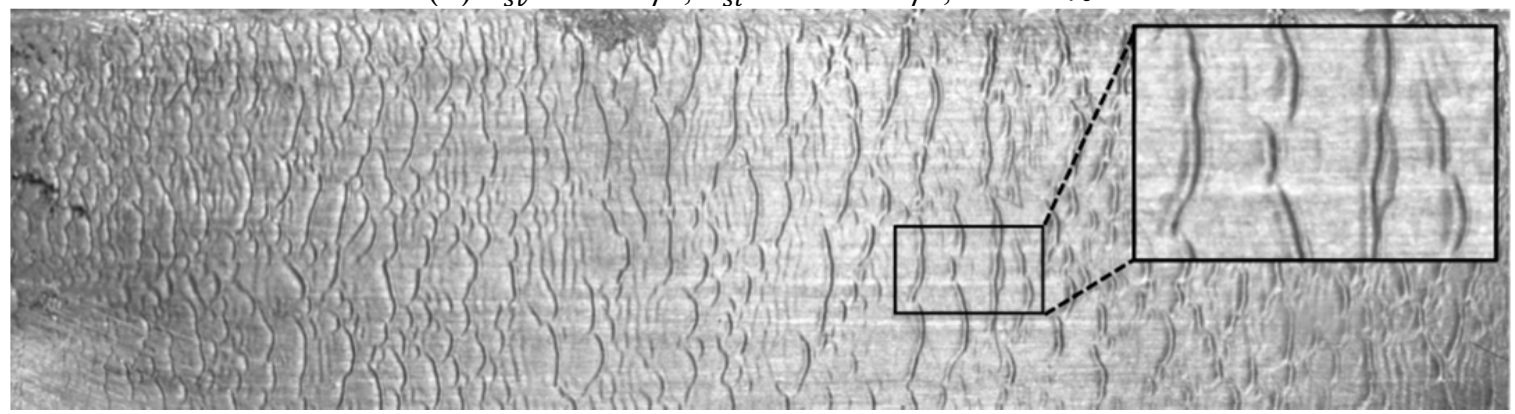

(d) $U_{s v}=13 \mathrm{~m} / \mathrm{s}, U_{s l}=0.027 \mathrm{~m} / \mathrm{s}, x=78 \%$

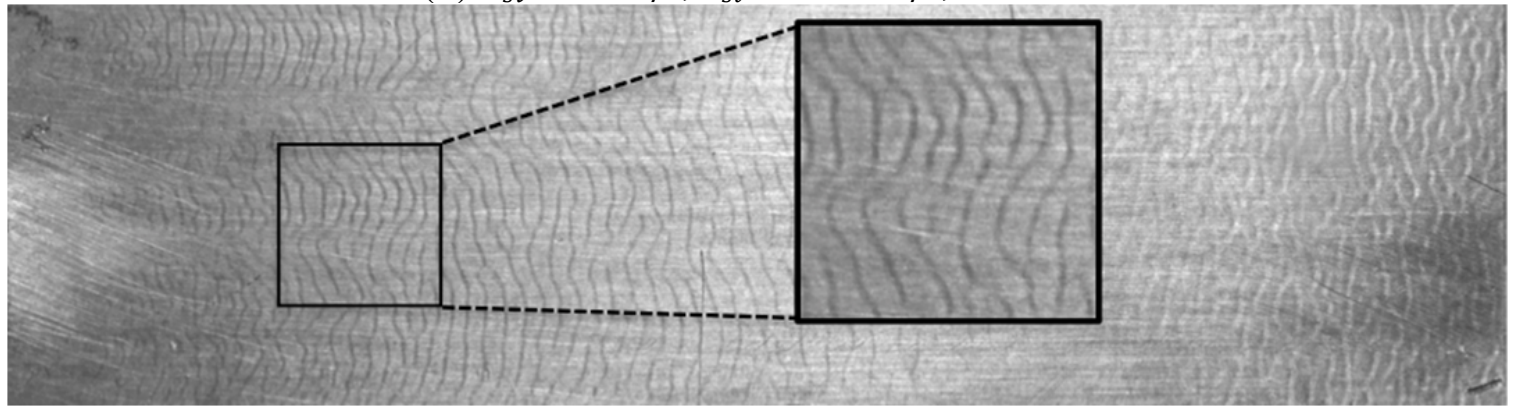

Fig. 4. Experimental adiabatic microgap visualization for $x=39-78 \%$, FC-72, $G=220 \mathrm{~kg} / \mathrm{m}^{2}$-s, and $\delta=184 \mu \mathrm{m}$ : (a) intermittent-to-annular transition, (b) 3D waves, (c) reduced amplitude 3-D waves, (d) continuous waves. Flow is from left to right. 
Table 3

Experimental flow quality, superficial velocities, average, and measured range of interfacial wavelengths for a mass flux of $220 \mathrm{~kg} / \mathrm{m}^{2}$-s.

\begin{tabular}{lllll}
\hline$x$ & $U_{s v}$ & $U_{s l}$ & $\lambda_{\text {avg }}$ & $\lambda_{\text {std.dev. }}$ \\
\hline $39 \%$ & $6.6 \mathrm{~m} / \mathrm{s}$ & $0.082 \mathrm{~m} / \mathrm{s}$ & $N / A$ & $N / A$ \\
$54 \%$ & $8.6 \mathrm{~m} / \mathrm{s}$ & $0.058 \mathrm{~m} / \mathrm{s}$ & $0.84 \mathrm{~mm}$ & $\pm 0.71 \mathrm{~mm}$ \\
$66 \%$ & $11 \mathrm{~m} / \mathrm{s}$ & $0.043 \mathrm{~m} / \mathrm{s}$ & $0.79 \mathrm{~mm}$ & $\pm 0.73 \mathrm{~mm}$ \\
$78 \%$ & $13 \mathrm{~m} / \mathrm{s}$ & $0.027 \mathrm{~m} / \mathrm{s}$ & $0.78 \mathrm{~mm}$ & $\pm 0.48 \mathrm{~mm}$ \\
\hline
\end{tabular}

(a) $U_{s v}=13 \mathrm{~m} / \mathrm{s}, U_{s l}=0.14 \mathrm{~m} / \mathrm{s}, x=43 \%$

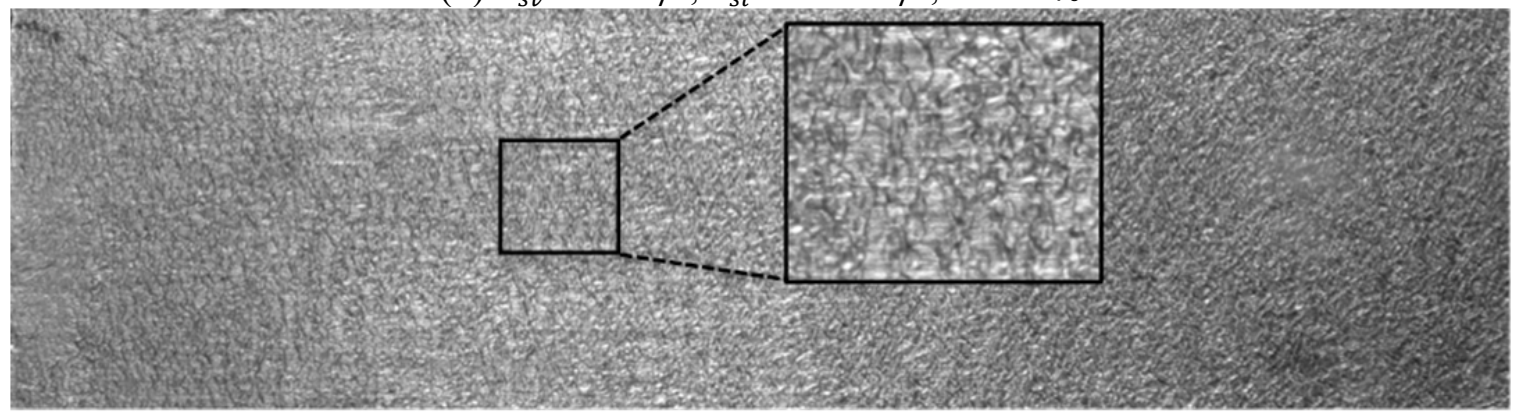

(b) $U_{s v}=20 \mathrm{~m} / \mathrm{s}, U_{s l}=0.071 \mathrm{~m} / \mathrm{s}, x=72 \%$

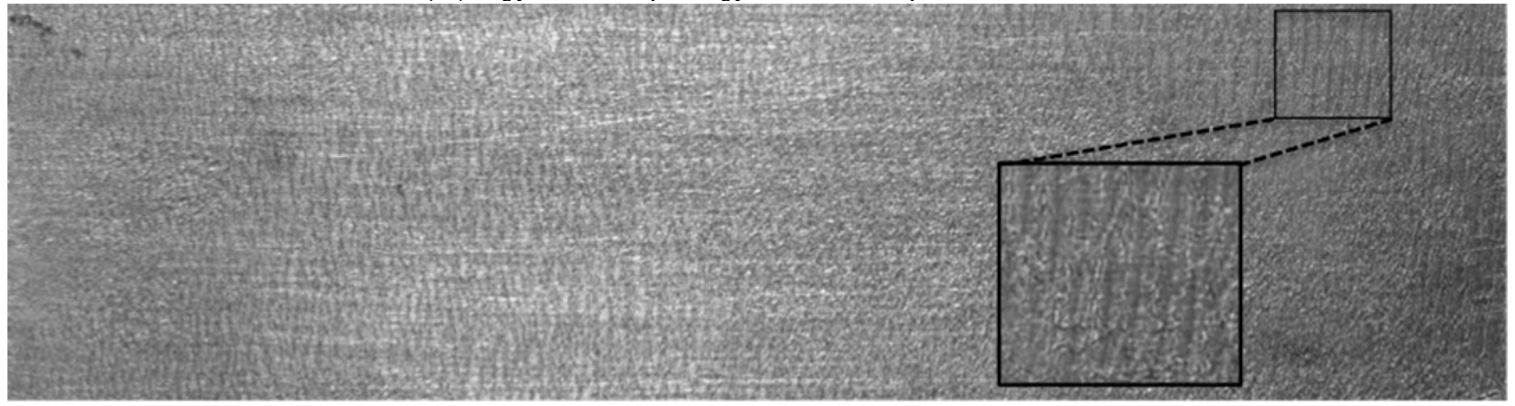

(c) $U_{s v}=24 \mathrm{~m} / \mathrm{s}, U_{s l}=0.035 \mathrm{~m} / \mathrm{s}, x=85 \%$

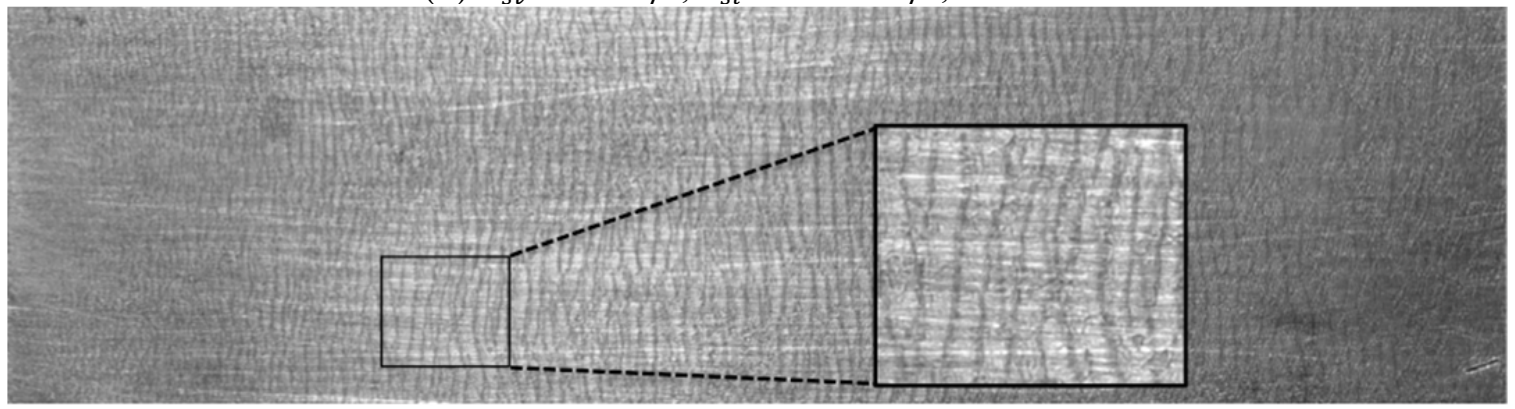

Fig. 5. Experimental adiabatic microgap visualization for $x=43-85 \%, F C-72, \mathrm{G}=420 \mathrm{~kg} / \mathrm{m}^{2}$-s, and $\delta=184 \mu \mathrm{m}$ : (a) chaotic structure, (b) chaotic to continuous wave transition, (c) continuous waves. Flow is from left to right. 
Table 4

Experimental flow quality, superficial velocities, and measured range of interfacial wavelengths for a mass flux of $420 \mathrm{~kg} / \mathrm{m}^{2}-\mathrm{s}$.

\begin{tabular}{lllll}
\hline$x$ & $U_{s v}$ & $U_{s l}$ & $\lambda_{\text {avg }}$ & $\lambda_{\text {std.dev. }}$ \\
\hline $43 \%$ & $13 \mathrm{~m} / \mathrm{s}$ & $0.14 \mathrm{~m} / \mathrm{s}$ & $0.40 \mathrm{~mm}$ & $0.26 \mathrm{~mm}$ \\
$56 \%$ & $16 \mathrm{~m} / \mathrm{s}$ & $0.11 \mathrm{~m} / \mathrm{s}$ & $0.37 \mathrm{~mm}$ & $0.28 \mathrm{~mm}$ \\
$72 \%$ & $20 \mathrm{~m} / \mathrm{s}$ & $0.071 \mathrm{~m} / \mathrm{s}$ & $0.34 \mathrm{~mm}$ & $0.24 \mathrm{~mm}$ \\
$85 \%$ & $24 \mathrm{~m} / \mathrm{s}$ & $0.035 \mathrm{~m} / \mathrm{s}$ & $0.23 \mathrm{~mm}$ & $0.12 \mathrm{~mm}$ \\
\hline
\end{tabular}

(a) $U_{s v}=15 \mathrm{~m} / \mathrm{s}, U_{s l}=0.24 \mathrm{~m} / \mathrm{s}, x=38 \%$

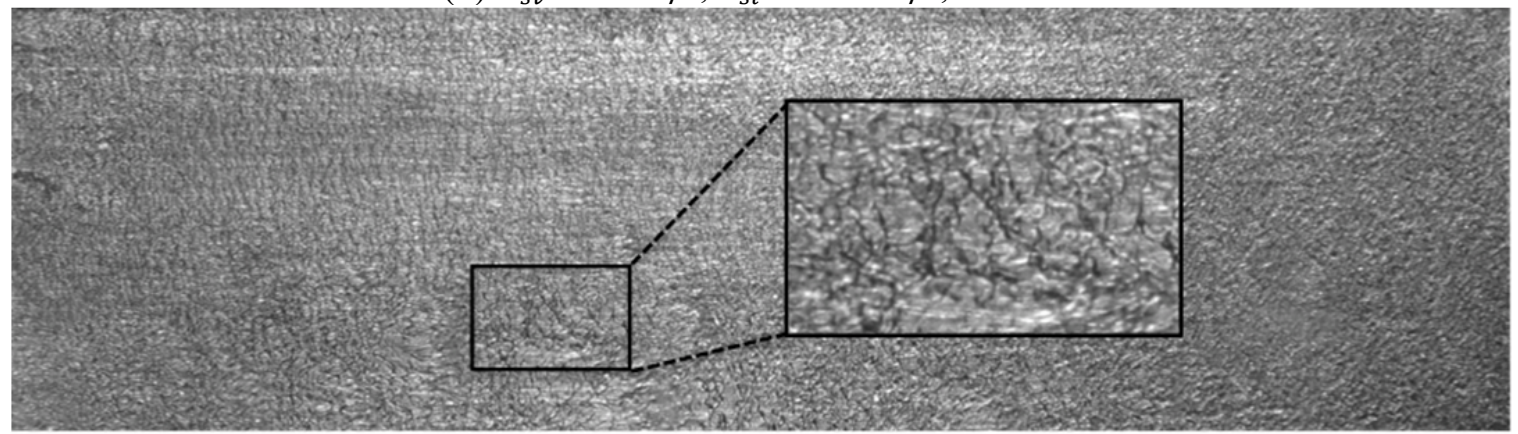

(b) $U_{s v}=21 \mathrm{~m} / \mathrm{s}, U_{s l}=0.18 \mathrm{~m} / \mathrm{s}, x=54 \%$

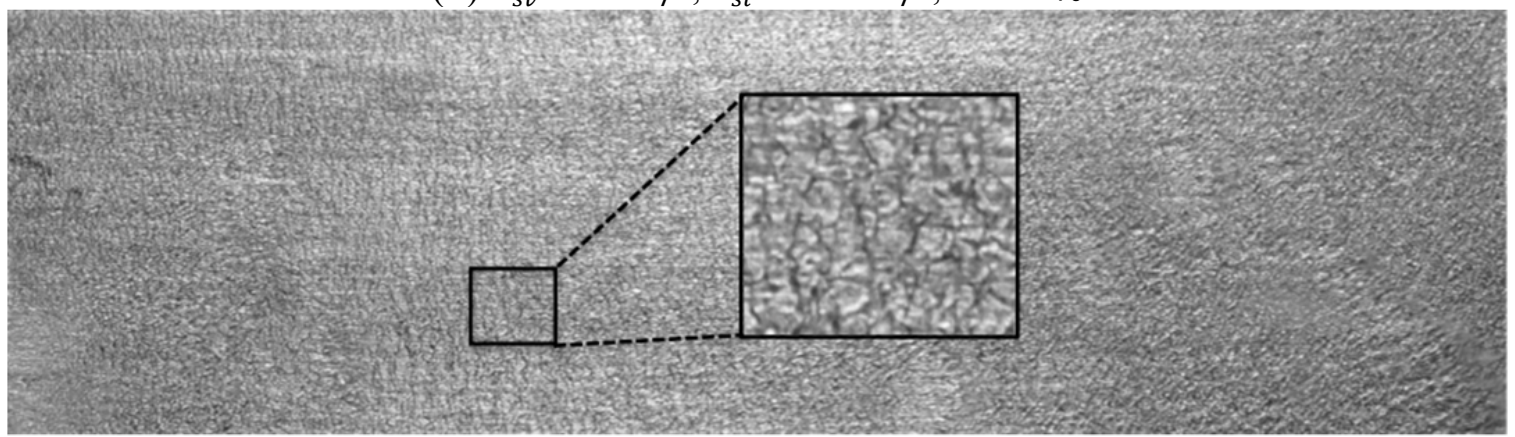

(c) $U_{s v}=25 \mathrm{~m} / \mathrm{s}, U_{s l}=0.12 \mathrm{~m} / \mathrm{s}, x=70 \%$

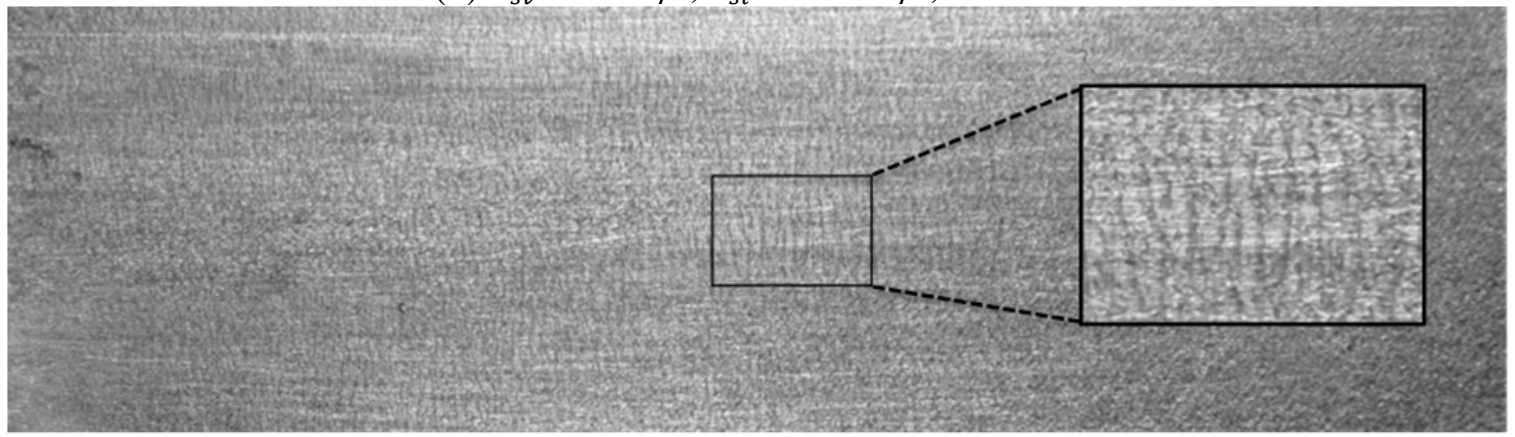

Fig. 6. Experimental adiabatic microgap visualization for $x=38-70 \%$, FC-72, $G=620 \mathrm{~kg} / \mathrm{m}^{2}$-s, and $\delta=184 \mu \mathrm{m}$ : (a) chaotic structure, (b) chaotic structure \& liquid bridges, (c) continuous waves. Flow is from left to right. 
Table 5

Experimental flow quality, superficial velocities, and measured range of interfacial wavelengths for a mass flux of $620 \mathrm{~kg} / \mathrm{m}^{2}-\mathrm{s}$.

\begin{tabular}{lllll}
\hline$x$ & $U_{s v}$ & $U_{s l}$ & $\lambda_{\text {avg }}$ & $\lambda_{\text {std.dev. }}$ \\
\hline $38 \%$ & $15 \mathrm{~m} / \mathrm{s}$ & $0.24 \mathrm{~m} / \mathrm{s}$ & $0.42 \mathrm{~mm}$ & $0.26 \mathrm{~mm}$ \\
$54 \%$ & $21 \mathrm{~m} / \mathrm{s}$ & $0.18 \mathrm{~m} / \mathrm{s}$ & $0.27 \mathrm{~mm}$ & $0.17 \mathrm{~mm}$ \\
$70 \%$ & $25 \mathrm{~m} / \mathrm{s}$ & $0.12 \mathrm{~m} / \mathrm{s}$ & $0.29 \mathrm{~mm}$ & $0.18 \mathrm{~mm}$ \\
$87 \%$ & $30 \mathrm{~m} / \mathrm{s}$ & $0.046 \mathrm{~m} / \mathrm{s}$ & $0.21 \mathrm{~mm}$ & $0.13 \mathrm{~mm}$ \\
\hline
\end{tabular}

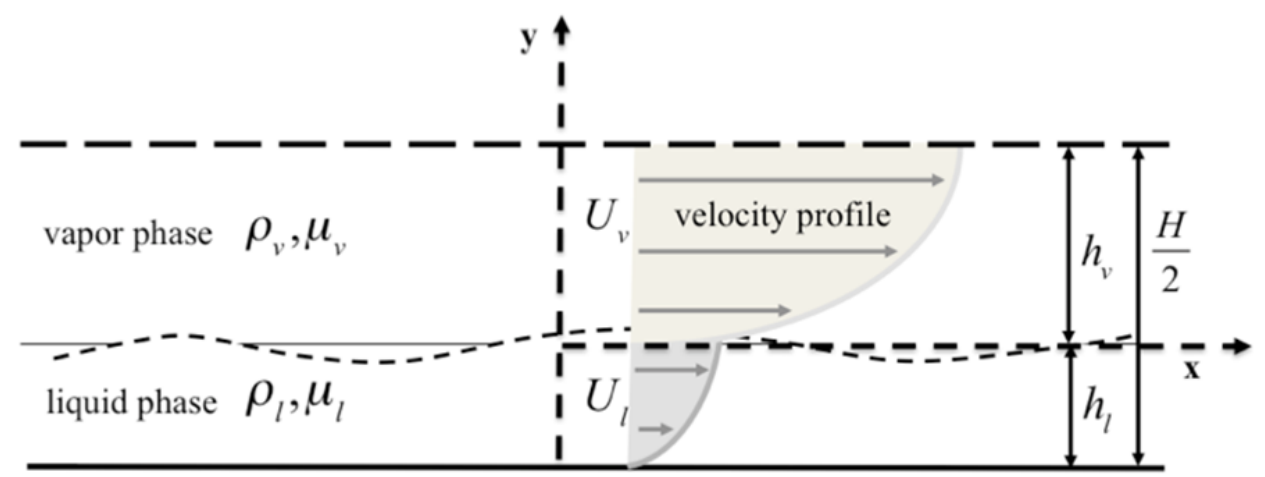

Fig. 7. Two-dimensional diagram of fully developed two-phase annular flow in a microgap.

Table 6

Flow parameters corresponding to various experimental conditions.

\begin{tabular}{ccc|ccc|cc|cc}
\hline $\begin{array}{c}G \\
\left(\mathrm{~kg} / \mathrm{m}^{2}-\mathrm{s}\right)\end{array}$ & $\begin{array}{c}x \\
(\%)\end{array}$ & $\alpha$ & $m$ & $r$ & $n$ & $R e$ & $W e$ & $R e_{I}$ & $\begin{array}{c}W e_{I} \\
\left(\times 10^{-3}\right)\end{array}$ \\
\hline 220 & 54 & 0.94 & 33 & 113 & 16 & 2701 & 51 & 2.11 & 5.00 \\
220 & 66 & 0.96 & 33 & 113 & 23 & 2729 & 51 & 1.00 & 1.62 \\
220 & 78 & 0.97 & 33 & 112 & 35 & 2746 & 50 & 0.47 & 0.57 \\
& & & & & & & & & \\
420 & 43 & 0.92 & 33 & 110 & 11 & 5024 & 177 & 8.63 & 57.3 \\
420 & 56 & 0.94 & 32 & 106 & 16 & 5120 & 176 & 4.11 & 18.2 \\
420 & 72 & 0.96 & 32 & 105 & 27 & 5185 & 173 & 1.66 & 4.94 \\
420 & 85 & 0.98 & 32 & 103 & 48 & 5195 & 171 & 0.39 & 0.59 \\
& & & & & & & & & \\
620 & 38 & 0.90 & 31 & 100 & 9 & 7196 & 351 & 19.0 & 219 \\
620 & 54 & 0.93 & 30 & 93 & 14 & 7413 & 348 & 8.28 & 68.1 \\
620 & 70 & 0.96 & 30 & 89 & 22 & 7510 & 337 & 2.62 & 11.0 \\
620 & 87 & 0.98 & 29 & 85 & 48 & 7519 & 325 & 0.59 & 1.05 \\
\hline
\end{tabular}




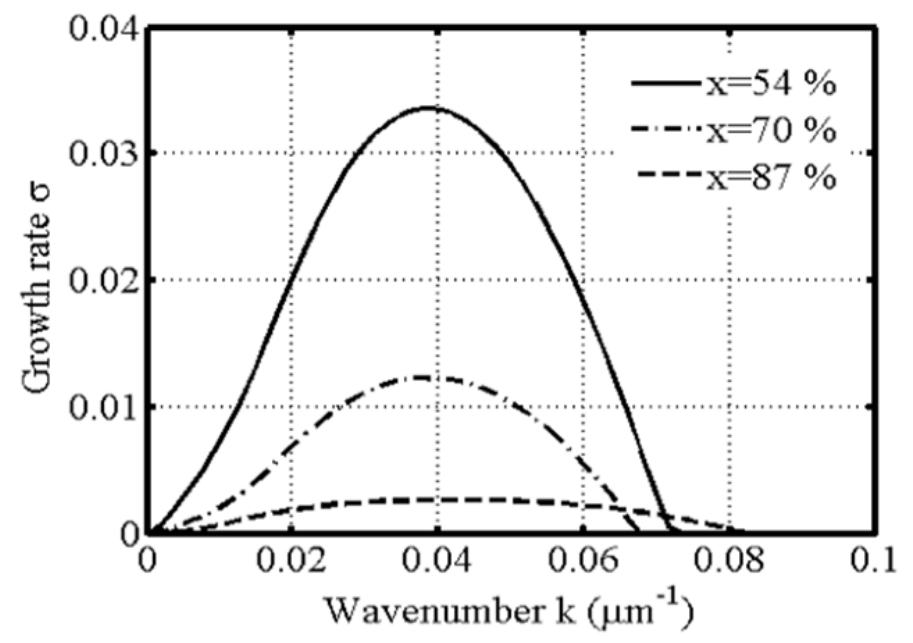

Fig. 8. Growth rate versus wavenumber $k$ for $G=620 \mathrm{~kg} / \mathrm{m}^{2}-\mathrm{s}, \mathrm{FC}-72$, and $\delta=184 \mu \mathrm{m}, x=54 \%$ (solid), $x=70 \%$ (dash-dot), $x=87 \%$ (dash).

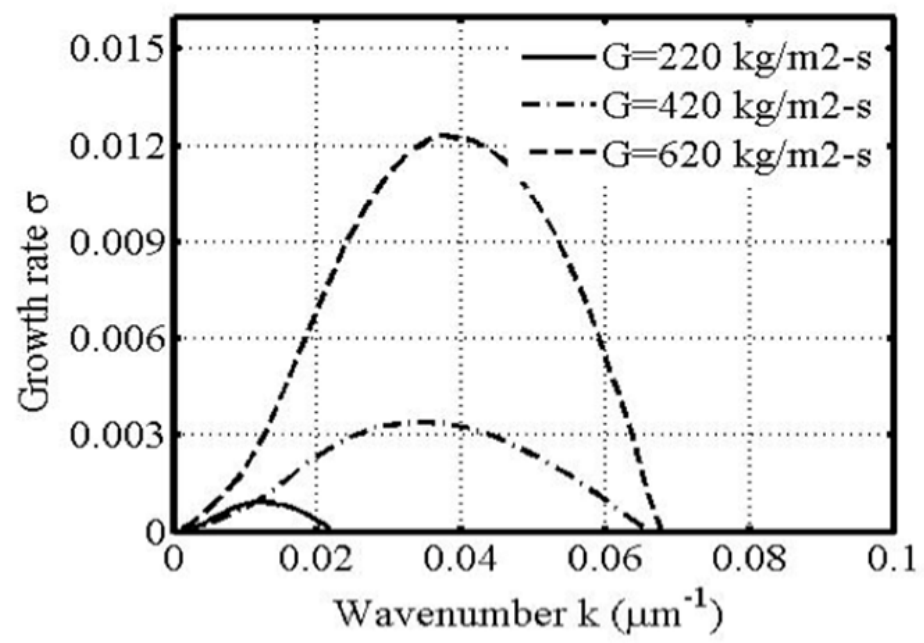

Fig. 9. Growth rate versus wavenumber $k$ for FC-72, and $\delta=184 \mu \mathrm{m} ; G=220 \mathrm{~kg} / \mathrm{m}^{2}$-s, $x=78 \%$ (solid), $G=420 \mathrm{~kg} / \mathrm{m}^{2}-\mathrm{s}, x=72 \%$ (dash-dot), $G=620 \mathrm{~kg} / \mathrm{m}^{2}-\mathrm{s}, x=70 \%$ (dash). 
(a)

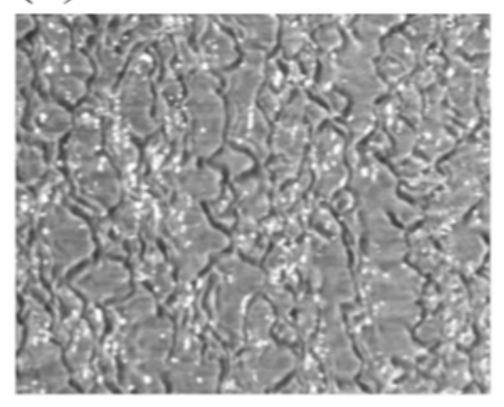

(b)

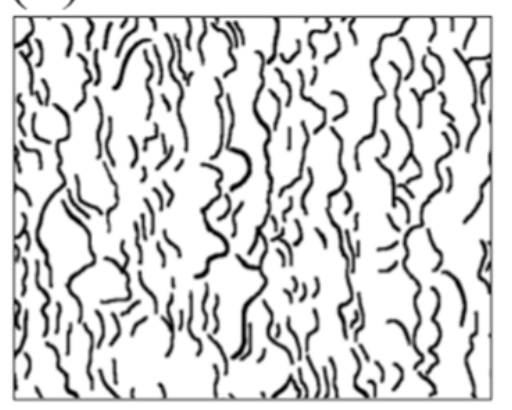

(c)

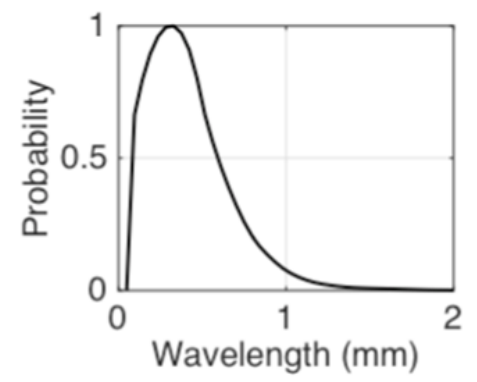

Fig. 10. Overview of the procedure used to experimentally measure interfacial wavelengths: (a) Original picture with direct illumination, (b) 2-bit image after image processing, and (c) wavelength distribution after Matlab processing. Data taken from $x=43 \%$ and $G=420 \mathrm{~kg} / \mathrm{m}^{2}$-s case.

Table 7

The relative difference between the experimental thermodynamic quality, $x$, defined by Eq. 1 , and the hydrodynamic quality, $x_{\alpha}$, defined by Eq. 20., an adjusted hydrodynamic quality, $x_{\widetilde{\alpha}}$, is defined in Eq. 21 to match $x$ by adjusting the layer thickness, $\tilde{\alpha}$.

\begin{tabular}{cc|ccc|cc}
\hline$G$ & $x$ & $\frac{\left|x-x_{\alpha}\right|}{x}$ & $\tilde{\alpha}$ & $\frac{|\tilde{\alpha}-\alpha|}{\alpha}$ & $\begin{array}{c}R e \\
\left(10^{3}\right)\end{array}$ & $\begin{array}{c}W e \\
\left(10^{2}\right)\end{array}$ \\
\hline 220 & 54 & 0.72 & 0.74 & 0.22 & 1.546 & 0.211 \\
220 & 66 & 0.44 & 0.78 & 0.19 & 1.878 & 0.294 \\
220 & 78 & 0.23 & 0.83 & 0.15 & 2.220 & 0.382 \\
420 & 43 & 1.17 & 0.69 & 0.25 & 2.294 & 0.486 \\
420 & 56 & 0.70 & 0.74 & 0.22 & 2.999 & 0.757 \\
420 & 72 & 0.36 & 0.79 & 0.17 & 3.811 & 1.118 \\
420 & 85 & 0.15 & 0.86 & 0.12 & 4.495 & 1.467 \\
620 & 38 & 1.41 & 0.66 & 0.26 & 2.975 & 0.794 \\
620 & 54 & 0.78 & 0.72 & 0.23 & 4.203 & 1.389 \\
620 & 70 & 0.39 & 0.78 & 0.18 & 5.338 & 2.094 \\
620 & 87 & 0.13 & 0.88 & 0.11 & 6.615 & 2.878 \\
\hline
\end{tabular}



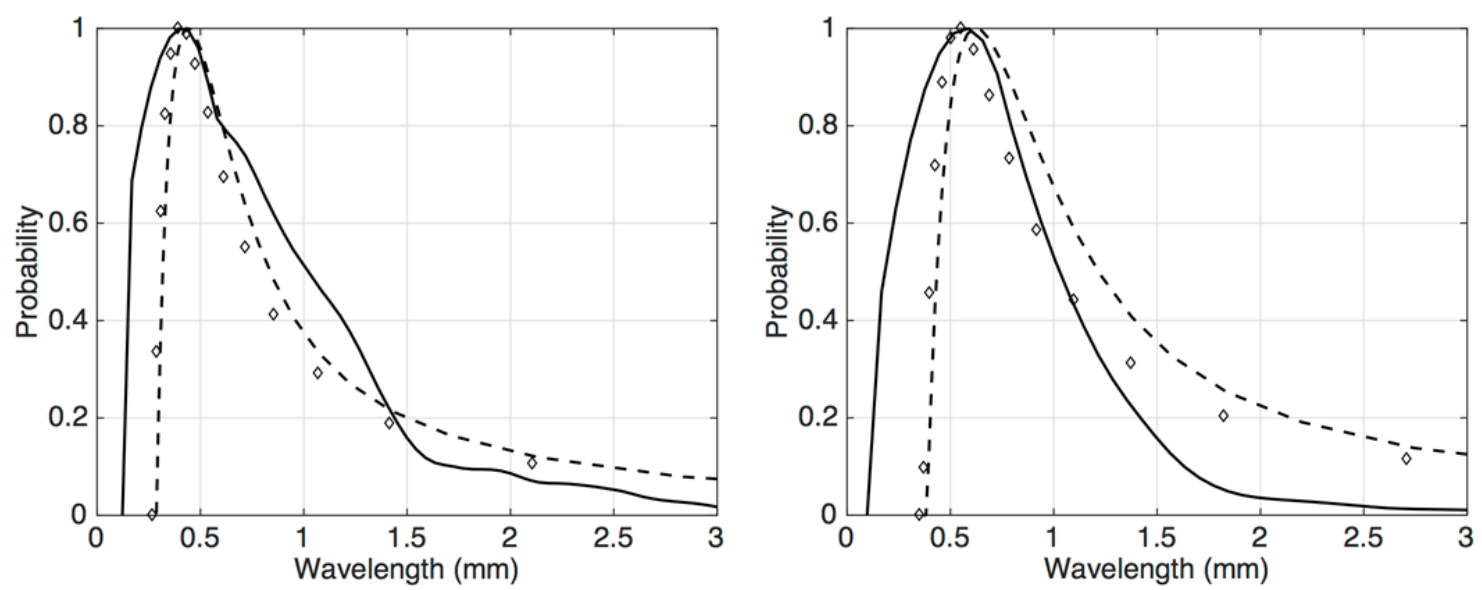

Fig. 11. Probability distribution of wavelengths for $G=220 \mathrm{~kg} / \mathrm{m}^{2}$-s: (left) $x=54 \%$ (right) $x=$ $66 \%$. Experimental data (solid line), linear stability results, data set 1 based on dimensionless parameters in Table 6 (dashed line) and data set 2 based on dimensionless parameters in Table 7 (symbols)
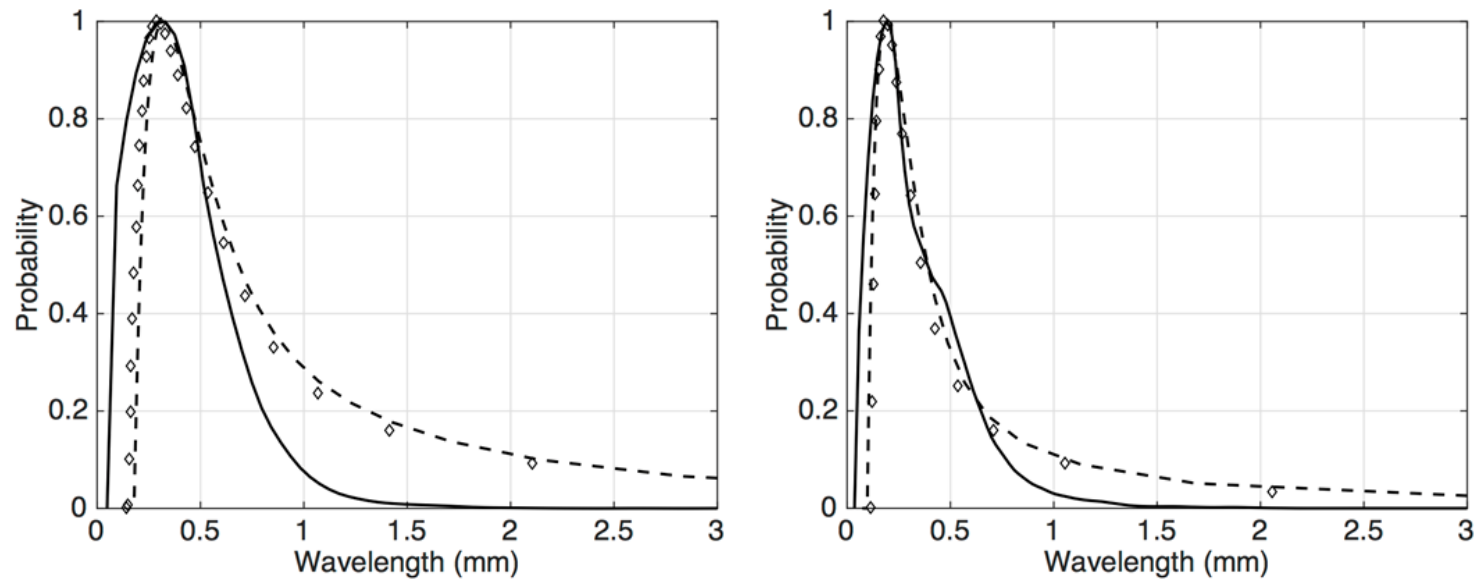

Fig. 12. Probability distribution of wavelengths for $G=420 \mathrm{~kg} / \mathrm{m}^{2}$-s: (left) $x=43 \%$ (right) $x=$ $72 \%$. Experimental data (solid line), linear stability results, data set 1 based on dimensionless parameters in Table 6 (dashed line) and data set 2 based on dimensionless parameters in Table 7 (symbols)
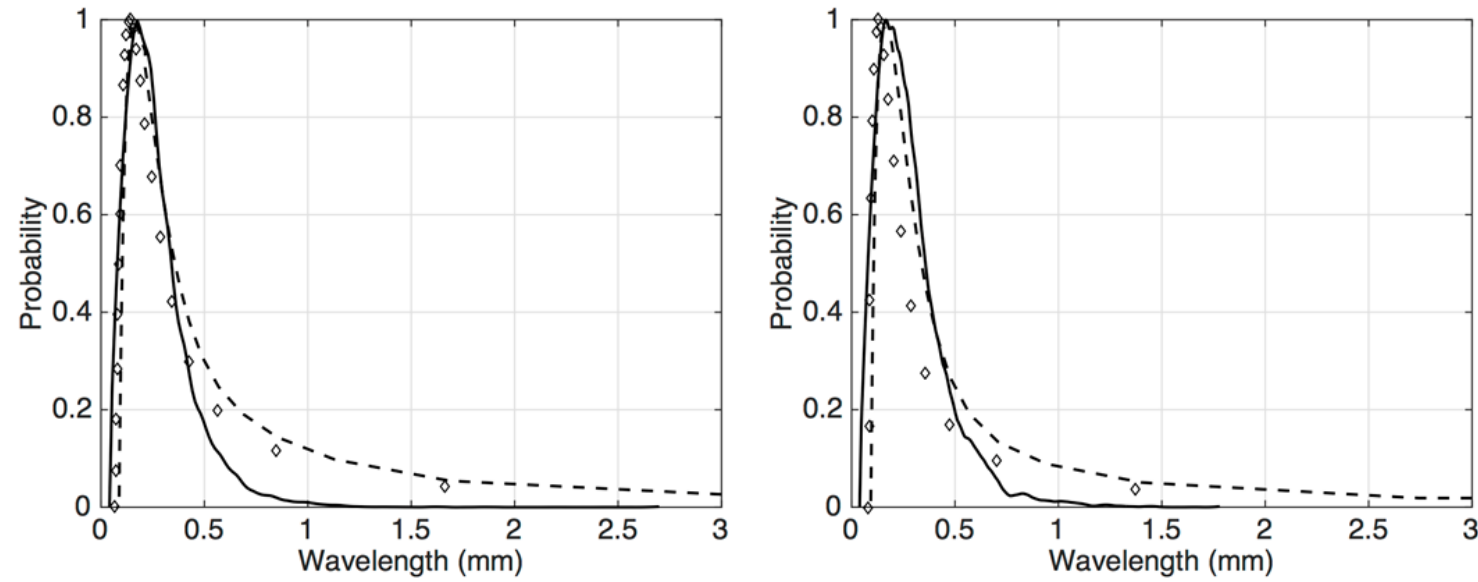

Fig. 13. Probability distribution of wavelengths for $G=620 \mathrm{~kg} / \mathrm{m}^{2}$-s: (left) $x=54 \%$ (right) $x=$ $70 \%$. Experimental data (solid line), linear stability results, data set 1 based on dimensionless parameters in Table 6 (dashed line) and data set 2 based on dimensionless parameters in Table 7 (symbols) 
Table 8:

Comparison of most probable experimental wavelengths with the most unstable wavelength predicted by linear stability analysis.

\begin{tabular}{ccccc}
\hline$G\left(\mathrm{~kg} / \mathrm{m}^{2}-\mathrm{s}\right)$ & $x(\%)$ & $\lambda_{m}$, Numerical $(\mathrm{mm})$ & $\bar{\lambda}_{m}$, Experimental $(\mathrm{mm})$ & $\%$ Error \\
\hline 220 & 54 & 0.430 & 0.40 & 7.02 \\
220 & 66 & 0.614 & 0.59 & 3.97 \\
220 & 78 & 0.505 & 0.51 & 0.81 \\
420 & 43 & 0.318 & 0.33 & 3.49 \\
420 & 56 & 0.138 & 0.14 & 0.79 \\
420 & 72 & 0.189 & 0.19 & 0.01 \\
420 & 85 & 0.171 & 0.19 & 11.1 \\
620 & 38 & 0.249 & 0.24 & 3.79 \\
620 & 54 & 0.171 & 0.17 & 0.59 \\
620 & 70 & 0.168 & 0.16 & 5.11 \\
620 & 87 & 0.151 & 0.15 & 0.61 \\
\hline
\end{tabular}

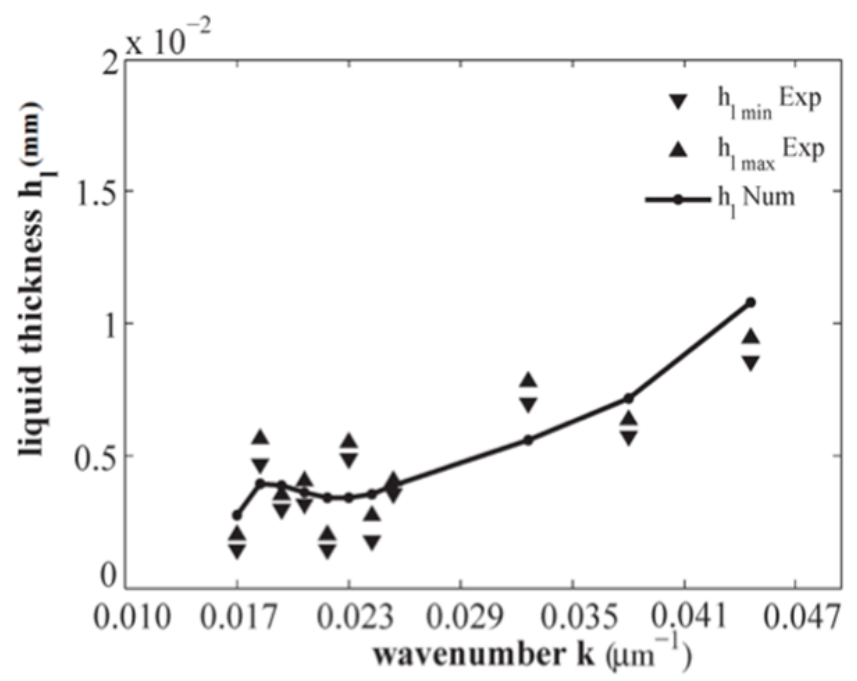

Fig. 14. Thickness of the liquid film $h_{l}(\mathrm{~mm}) \mathrm{h}_{\mathrm{l}}$ versus the wavenumber $k\left(\mu \mathrm{m}^{-1}\right)$ for different experimental conditions listed in Table 6 in the manuscript. 


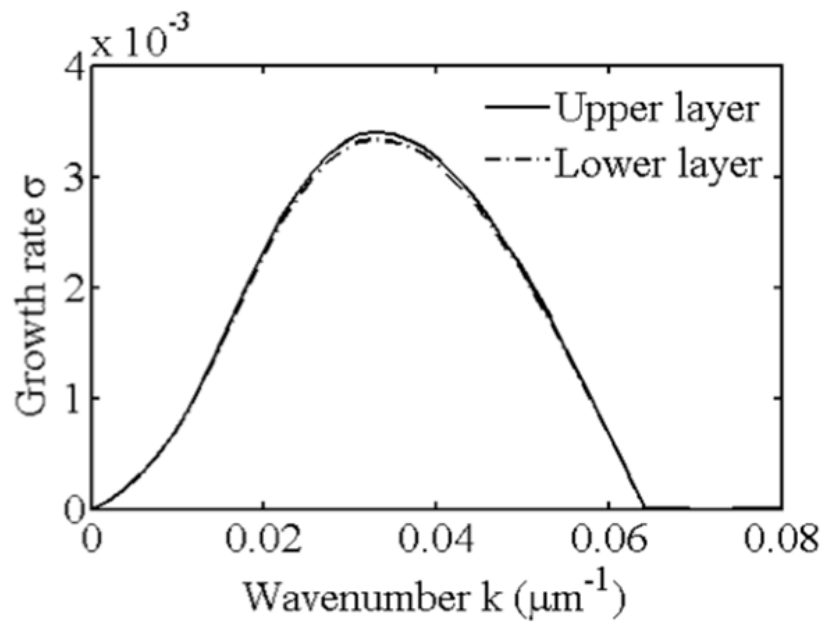

Fig. 15. The growth rates versus the wavenumber for the upper and lower layers for the experimental case of $\mathrm{G}=420 \mathrm{~kg} / \mathrm{m}^{2} \mathrm{~s}, r=105, m=32, n=26$ and $E o=0.0085$. The upper layer results are obtained by using a negative value for the gravitational constant, $g$. 